\title{
Selection rules for Single-Chain-Magnet behavior in non-collinear Ising systems
}

\author{
Alessandro Vindigni年 and Maria Gloria Pini² \\ ${ }^{1}$ Laboratorium für Festkörperphysik, Eidgenössische Technische Hochschule Zürich, CH-8093 Zürich, Switzerland \\ ${ }^{2}$ Istituto dei Sistemi Complessi, Consiglio Nazionale delle Ricerche, \\ Via Madonna del Piano 10, I-50019 Sesto Fiorentino (FI), Italy
}

(Dated: November 2, 2018)

\begin{abstract}
The magnetic behavior of molecular Single-Chain Magnets is investigated in the framework of a one-dimensional Ising model with single spin-flip Glauber dynamics. Opportune modifications to the original theory are required in order to account for reciprocal non-collinearity of local anisotropy axes and the crystallographic (laboratory) frame. The extension of Glauber's theory to the case of a collinear Ising ferrimagnetic chain is also discussed. Within this formalism, both the dynamics of magnetization reversal in zero field and the response of the system to a weak magnetic field, oscillating in time, are studied. Depending on the geometry, selection rules are found for the occurrence of slow relaxation of the magnetization at low temperatures, as well as for resonant behavior of the a.c. susceptibility as a function of temperature at low frequencies. The present theory applies successfully to some real systems, namely Mn-, Dy-, and Co-based molecular magnetic chains, showing that Single-Chain-Magnet behavior is not only a feature of collinear ferro- and ferrimagnetic, but also of canted antiferromagnetic chains.
\end{abstract}

PACS numbers: 75.10.-b, 75.10.Pq, 75.50.Xx, 75.60.Jk

\section{INTRODUCTION}

Slow dynamics of the magnetization reversal is a crucial requirement for potential applications of SingleChain Magnets (SCM's) $1,2.3$, and nanowires in general, in magnetic-memory manufacture. For nanowires with a biaxial anisotropy, provided that their length is much greater than the cross section diameter but smaller than exchange length, this phenomenon is governed by thermal nucleation and propagation of soliton-antisoliton pairs; the associated characteristic time is expected to follow an Arrhenius law $\stackrel{4.5}{ }$. For genuine one dimensional (1D) Ising systems with single spin-flip stochastic dynamics, a slow relaxation of the magnetization was first predicted by Glauber ${ }^{6}$ in 1963 . Through Glauber's approach, many physical systems were investigated, ranging from dielectrics $7,8,9$ to polymers $7,10,11$. More fundamentally, this model has been employed to justify the use of the Kohlrauch-Williams-Watts function 10,12 (stretched exponential) to fit the relaxation of generalized 1D spin systems. Also the universality issue of the dynamic critical exponent $13,14,15,16,17$ of the Ising model ${ }^{18}$, as well as strongly out-of-equilibrium processes (magnetization reversal ${ }^{19}$, facilitated dynamics $\frac{19}{}$, etc.) have been studied moving from the basic ideas proposed by Glauber.

In this paper, single spin-flip Glauber dynamics is used to investigate theoretically the slow dynamics of the magnetization reversal in molecular magnetic systems. In particular, we extend Glauber's theory $\underline{\underline{6}}$ to the Ising collinear ferrimagnetic chain, as well as to the case of a chain in which reciprocal non-collinearity of local anisotropy axes and the crystallographic (laboratory) frame is encountered. Such extensions are motivated by the fact that (i) in molecular-based realizations of SCM's, antiferromagnetic coupling typically has a larger intensity than the ferromagnetic one; in fact, the overlapping of magnetic orbitals, which implies antiferromagnetic exchange interaction between neighboring spins, can be more easily obtained than the orthogonality condition, leading to ferromagnetism 20.21 ,22; (ii) non-collinearity between local anisotropy axes and the crystallographic (laboratory) frame takes place quite often in molecular spin chains. Besides magnetization reversal, the dynamic response of the system to a weak magnetic field, oscillating in time at frequency $\omega$, is also studied. Depending on the specific experimental geometry, selection rules are found for the occurrence of resonant behavior of the a.c. susceptibility as a function of temperature (stochastic resonance) at low frequencies, as well as for slow relaxation of the magnetization in zero field at low temperatures.

The paper is organized as follows. In Sect. II we extend Glauber's theory $\underline{\underline{6}}$, originally formulated for a chain of identical and collinear spins, to the more general model of a chain with non-collinear spins, possibly with Landé factors that vary from site to site. In Sect. III we use two different theoretical methods (the Generating Functions approach and the Fourier Transform approach) to investigate the relaxation of the magnetization after removal of an external static magnetic field, starting from two different initial conditions: fully saturated or partially saturated. In Sect. IV we calculate, in a linear approximation, the magnetic response of the system to an oscillating magnetic field. For a chain of $N$ spins, the a.c. susceptibility is expressed as the superposition of $N$ contributions, each characterized by its time scale; through a few simple examples, we show that, depending on the geometry of the system (i.e., the relative orientations of the local easy anisotropy axes and of the applied field), different time scales can be selected, possibly giving rise, for low frequencies, to a resonant peak in the temperaturedependence of the complex magnetic susceptibility. In Sect. V we show that the theory provides a satisfactory 
account for the SCM behavior experimentally observed in some magnetic molecular chain compounds, characterized by dominant antiferromagnetic exchange interactions and non-collinearity between spins. Finally, in Sect. VI, the conclusions are drawn and possible forthcoming applications are also discussed.

\section{THE NON-COLLINEAR ISING-GLAUBER MODEL}

In a celebrated paper ${ }^{6}$, Glauber introduced, in the usual 1D Ising model ${ }^{18}$, a stochastic dependence on the time variable $t$ : i.e., the state of a spin lying on the $k$ th lattice site was represented by a two-valued stochastic function $\sigma_{k}(t)$

$$
\mathcal{H}_{I}=-\sum_{k=1}^{N}\left(J_{I} \sigma_{k} \sigma_{k+1}+g \mu_{B} \mathrm{H} e^{-i \omega t} \sigma_{k}\right), \quad \sigma_{k}(t)= \pm 1 .
$$

$J_{I}$ is the exchange coupling constant, that favors nearest neighboring spins to lie parallel $\left(J_{I}>0\right.$, ferromagnetic exchange) or antiparallel $\left(J_{I}<0\right.$, antiferromagnetic exchange); $g$ is the Landé factor of each spin, and $\mu_{B}$ the Bohr magneton. In the original paper ${ }^{6}$ a $1 \mathrm{D}$ lattice of equivalent and collinear spins was studied; there the response to a time-dependent magnetic field $\mathrm{H}(t)$, applied parallel to the axis of spin quantization and oscillating with frequency $\omega$, as in typical a.c. susceptibility experiments, was also considered.

In order to investigate the phenomena of slow relaxation (for $\mathrm{H}=0$ ) and resonant behavior of the a.c. susceptibility (for $\mathrm{H} \neq 0$ ) in molecular SCM's, we are going to generalize the Glauber model (1) accounting for noncollinearity of local anisotropy axes and crystallographic (laboratory) frame. To this aim, we adopt the following model Hamiltonian

$$
\mathcal{H}=-\sum_{k=1}^{N}\left(J_{I} \sigma_{k} \sigma_{k+1}+G_{k} \mu_{B} \mathrm{H} e^{-i \omega t} \sigma_{k}\right), \quad \sigma_{k}(t)= \pm 1 .
$$

$J_{I}$ is an effective Ising exchange coupling that can approximately be related to the Hamiltonian parameters of a real $\mathrm{SCM}^{23,24}$ : see later on the discussion in Section V. Like in the usual Ising-Glauber collinear model (1), the spins in Eq. (2) are described by classical, onecomponent vectors that are allowed to take two integer values $\sigma_{k}(t)= \pm 1$, but now the magnetic moments may be oriented along different directions, $\hat{\mathbf{z}}_{k}$, varying from site to site. Within this scheme, the Landé tensor of a spin on the $k$-th lattice site has just a non-zero component, $g_{k}^{\|}$, along the local easy anisotropy direction $\hat{\mathbf{z}}_{k}$. Denoting by $\hat{\mathbf{e}}_{\mathrm{H}}$ the direction of the oscilla.ting magnetic field, $\mathbf{H}(t)=\mathrm{H} e^{-i \omega t} \hat{\mathbf{e}}_{\mathrm{H}}$, we define the generalized Landé factor $G_{k}$ appearing in Eq. (2) as

$$
G_{k}=g_{k}^{\|} \hat{\mathbf{z}}_{k} \cdot \hat{\mathbf{e}}_{\mathrm{H}}
$$

i.e., a scalar quantity that varies from site to site. Following Glauber ${ }^{6}$, we define the single spin expectation value $s_{k}(t)=\left\langle\sigma_{k}\right\rangle_{t}$, where brackets denote a proper ensemble average, and the stochastic magnetization along the direction of the applied field

$$
\langle M\rangle_{t}=\mu_{B} \sum_{k=1}^{N} G_{k}\left\langle\sigma_{k}\right\rangle_{t}=\mu_{B} \sum_{k=1}^{N} G_{k} s_{k}(t) .
$$

The basic equation of motion of the Glauber model ${ }^{12,14}$ reads

$$
\frac{d}{d t} s_{k}(t)=-2\left\langle\sigma_{k} w_{\sigma_{k} \rightarrow-\sigma_{k}}\right\rangle,
$$

in which $w_{\sigma_{k} \rightarrow-\sigma_{k}}$ represents the probability per unit time to reverse the $k$-th spin, through the flip $+\sigma_{k} \rightarrow$ $-\sigma_{k}$. For a system of $N$ coupled spins, this probability is affected by the interaction with the other spins, with the thermal bath and, possibly, with an external magnetic field. Among all possible assumptions for the transition probability $w_{\sigma_{k} \rightarrow-\sigma_{k}}$ as a function of the $N+1$ variables $11,19,25,26 \quad\left\{\sigma_{1}, \ldots, \sigma_{k}, \ldots, \sigma_{N}, t\right\}$, again following Glauber ${ }^{6}$ we require $w_{\sigma_{k} \rightarrow-\sigma_{k}}$ to be independent of time and to depend only on the configuration of the two nearest neighbors of the $k$-th spin. In zero field, these requirements are fulfilled by

$$
w_{\sigma_{k} \rightarrow-\sigma_{k}}^{\mathrm{H}=0}=\frac{1}{2} \alpha\left[1-\frac{1}{2} \gamma \sigma_{k}\left(\sigma_{k-1}+\sigma_{k+1}\right)\right]
$$

while in the presence of an external field

$$
w_{\sigma_{k} \rightarrow-\sigma_{k}}^{\mathrm{H}}=w_{\sigma_{k} \rightarrow-\sigma_{k}}^{\mathrm{H}=0}\left(1-\delta_{k} \sigma_{k}\right),
$$

is usually chosen; the attempt frequency $\frac{1}{2} \alpha$ (i.e., the probability per unit time to reverse an isolated spin) remains an undetermined parameter of the model; $\gamma$ accounts for the effect of the nearest neighbors; the parameters $\delta_{k}$ have the role of stabilizing the configuration in which the $k$-th spin is parallel to the field, and destabilizing the antiparallel configuration. Thanks to the particular choices (6) and (7) for the transition probability, by imposing the Detailed Balance conditions ${ }^{6}$ it is possible to express $\gamma$ and $\delta_{k}$ as functions of the parameters in the spin Hamiltonian (2)

$$
\gamma=\tanh \left(2 \beta J_{I}\right), \quad \delta_{k}=\tanh \left(\beta G_{k} \mu_{B} \mathrm{H}\right) .
$$

where $\beta=\frac{1}{k_{B} T}$ is the inverse temperature in units of Boltzmann's constant. Another advantage of Glauber's choices (6) and (7) is that the equation of motion (5) takes a simple form. In particular, for $\mathrm{H}=0$, Eq. (5) with the choice (6) becomes

$$
\frac{d \underline{s}(t)}{d t}=-\alpha \mathbf{A} \underline{s}(t),
$$

where $\underline{s}(t)$ denotes the vector of single-spin expectation values $\left\{s_{1}(t), s_{2}(t), \cdots, s_{N}(t)\right\}$ and $\mathbf{A}$ is a square $N \times N$ 
symmetric matrix, whose non-zero elements are $\mathrm{A}_{k, k}=1$ and $\mathrm{A}_{k, k-1}=\mathrm{A}_{k, k+1}=-\frac{\gamma}{2}$, with $\mathrm{A}_{1, N}=\mathrm{A}_{N, 1}=-\frac{\gamma}{2}$ if periodic boundary conditions are assumed for the $N$-spin chain. A closed solution of this set of first-order differential equations can be obtained expressing the expectation value of each spin, $s_{k}(t)$, in terms of its spatial Fourier Transform (FT) $\widetilde{s}_{q}$

$$
s_{k}(t)=\sum_{q} \widetilde{s}_{q} e^{i q k} e^{-\lambda_{q} t} .
$$

Substituting Eq. (10) into Eq. (9), one readily obtains the dispersion relation

$$
\lambda_{q}=\alpha(1-\gamma \cos q), \quad q=\frac{2 \pi}{N} n
$$

with $n=0,1, \ldots, N-127$. For ferromagnetic coupling $\left(J_{I}>0\right.$, hence $\left.\gamma>0\right)$ the smallest eigenvalue $\lambda_{q=0}=\alpha(1-\gamma)$ occurs for $n=0$, independently of the number of spins $N$ in the chain. For antiferromagnetic coupling $\left(J_{I}<0\right.$, hence $\left.\gamma<0\right)$ and $N$ even, the smallest eigenvalue $\lambda_{q=\pi}=\alpha(1-|\gamma|)$ occurs for $n=\frac{N}{2}$; while in the case of $N$ odd, the smallest eigenvalue corresponds to $\alpha\left[1-|\gamma| \cos \left(\frac{\pi}{N}\right)\right]$, thus depending on the number of spins in the antiferromagnetic chain ${ }^{28}$. The characteristic time scales of the system, $\tau_{q}$, are given by

$$
\tau_{q}=\frac{1}{\lambda_{q}}=\frac{1}{\alpha(1-\gamma \cos q)} .
$$

At finite temperatures the characteristic times $\tau_{q}$ are finite because $|\gamma|<1$; for $T \rightarrow 0$ one has that $1-|\gamma|$ vanishes irrespectively of the sign of $J_{I}$, because $\gamma \rightarrow \frac{J_{I}}{\left|J_{I}\right|}=$ \pm 1 . Thus, for $\mathrm{H}=0$, there is one diverging time scale in the $T \rightarrow 0$ limit: $\tau_{q=0}$ for ferromagnetic coupling and $\tau_{q=\pi}$ for antiferromagnetic coupling (and even $N$ ). In the presence of a non-zero, oscillating field $\mathrm{H}(t)=\mathrm{H} e^{-i \omega t}$, the equation of motion (5) with the choice (7) takes a form (see Eq. 24 in Section IV later on) which can still be solved, though in an approximate way ${ }^{\underline{6}}$, for a sufficiently weak intensity of the applied magnetic field.

\section{RELAXATION OF THE MAGNETIZATION IN ZERO FIELD}

The original Glauber model was formulated for a chain of collinear spins with the same Landé factors: i.e., in Eq. (2) one has $G_{k}=g, \forall k=1, \cdots, N$. Assuming that the system has been fully magnetized by means of a strong external field, one can study how the system evolves if the field is removed abruptly. This corresponds to take a fully saturated initial condition

$$
s_{k}(0)=1, \quad \forall k \text {. }
$$

In ferrimagnetic chains, on the other hand, a "partial" saturation can be reached, provided the antiferromagnetic coupling $\left(J_{I}<0\right)$ between nearest neighbors is "strong enough", in a sense that will be clarified later on. In fact, if the Landé factors for odd and even sites are not equal $\left(g_{o} \neq g_{e}\right)$, through the application of an opportune field the sample can be prepared in a configuration with

$$
\begin{cases}s_{k}(0)=+1, & \text { for } k=2 r+1 \quad(k \text { odd }) \\ s_{k}(0)=-1, & \text { for } k=2 r \quad(k \text { even }) .\end{cases}
$$

With respect to the case considered by Glauber, it is convenient to separate explicitly the expectation values of the odd sites, $s_{2 r+1}(t)$, from those of the even sites, $s_{2 r}(t)$. Thus, for $\mathrm{H}=0$, the set of $N$ equations of motion (9) can be rewritten as

$$
\left\{\begin{array}{l}
\frac{d}{d t} s_{2 r}=-\alpha\left[s_{2 r}+\frac{1}{2} \gamma\left(s_{2 r+1}+s_{2 r-1}\right)\right] \\
\frac{d}{d t} s_{2 r+1}=-\alpha\left[s_{2 r+1}+\frac{1}{2} \gamma\left(s_{2 r}+s_{2 r-2}\right)\right]
\end{array}\right.
$$

In the following, the solutions of (15) will be found using two different approaches that yield identical results.

\section{A. The Generating Function approach}

The Generating Function approach, which closely follows the original Glauber's paper, is exposed in detail in Appendix. Here, in order to distinguish between the ferromagnetic and ferrimagnetic relaxations, we specialize the general solution, Eq. (A5) and Eq. (A6), to the two different kinds of initial conditions, Eq. (13) and Eq. (14). In both cases, we will assume that the exchange coupling $J_{I}$ is negative. The "partially saturated" configuration, Eq. (14), reflects a typical experimental situation, in which the antiferromagnetic coupling is much bigger $\left(J_{I} \approx 100 \div 1000 \mathrm{~K}\right)$ than the Zeeman energy associated with accessible magnetic fields. On the other hand, the initial configuration with all the spins aligned in the same direction, Eq. (13), clearly reflects the experimental situation of a fully saturated sample. This condition is easily obtained for ferromagnetic coupling $\left(J_{I}>0\right)$, while it may require very strong fields (eventually unaccessible) for antiferromagnetic coupling $\left(J_{I}<0\right)$.

Let us start from the saturated configuration, Eq. (13). Substituting the initial condition $s_{k}(0)=1$ for all $k$ in both (A5) and (A6), we obtain

$$
\left\{\begin{array}{l}
s_{2 r}(t)=e^{-\alpha t} \sum_{m=-\infty}^{+\infty}\left[\mathcal{I}_{2(r-m)}(\gamma \alpha t)+\mathcal{I}_{2(r-m)-1}(\gamma \alpha t)\right] \\
s_{2 r+1}(t)=e^{-\alpha t} \sum_{m=-\infty}^{+\infty}\left[\mathcal{I}_{2(r-m)}(\gamma \alpha t)+\mathcal{I}_{2(r-m)+1}(\gamma \alpha t)\right] .
\end{array}\right.
$$

Hence, exploiting the property (A4) of the Bessel functions (taking $y=1$ ), and redefining the sums by a unique index $j$, we get

$$
\left\{\begin{array}{l}
s_{2 r}(t)=e^{-\alpha t} \sum_{j=-\infty}^{+\infty} \mathcal{I}_{j}(\gamma \alpha t)=e^{-\alpha(1-\gamma) t} \\
s_{2 r+1}(t)=e^{-\alpha t} \sum_{j=-\infty}^{+\infty} \mathcal{I}_{j}(\gamma \alpha t)=e^{-\alpha(1-\gamma) t}
\end{array}\right.
$$


This means that, starting with all the spins aligned in the same direction, each spin expectation value (both on even and odd sites) decays obeying a mono-exponential law with relaxation time $\tau_{q=0}=[\alpha(1-\gamma)]^{-1}$, which is just the characteristic time scale obtained as the inverse of the dispersion relation $\lambda_{q}$ with zero wave number $q=0$, see Eq. (11). Notice that $\tau_{q=0}$ can diverge for $T \rightarrow 0$ only in the case of ferromagnetic coupling, $J_{I}>0(\gamma>0)$.

Let us now consider the partially saturated configuration, Eq. (14), in which $s_{k}(0)=1$ for $k$ odd and $s_{k}(0)=-1$ for $k$ even. Substituting these initial conditions in both (A5) and (A6), we obtain

$$
\left\{\begin{array}{l}
s_{2 r}(t)=-e^{-\alpha t} \sum_{m=-\infty}^{+\infty}\left[\mathcal{I}_{2(r-m)}(\gamma \alpha t)-\mathcal{I}_{2(r-m)-1}(\gamma \alpha t)\right] \\
s_{2 r+1}(t)=e^{-\alpha t} \sum_{m=-\infty}^{+\infty}\left[\mathcal{I}_{2(r-m)}(\gamma \alpha t)-\mathcal{I}_{2(r-m)+1}(\gamma \alpha t)\right]
\end{array}\right.
$$

and, still exploiting the property (A4) (but now for $y=$ -1 ), we get

$$
\left\{\begin{array}{l}
s_{2 r}(t)=-e^{-\alpha t} \sum_{j=-\infty}^{+\infty}(-1)^{j} \mathcal{I}_{j}(\gamma \alpha t)=-e^{-\alpha(1+\gamma) t} \\
s_{2 r+1}(t)=e^{-\alpha t} \sum_{j=-\infty}^{+\infty}(-1)^{j} \mathcal{I}_{j}(\gamma \alpha t)=e^{-\alpha(1+\gamma) t}
\end{array}\right.
$$

Also in this case all the spins of the system relax with a mono-exponential law, but now the relaxation time is $\tau_{q=\pi}=[\alpha(1+\gamma)]^{-1}$, which corresponds to the inverse of the eigenvalue $\lambda_{q}$ with wave number $q=\pi$, see Eq. (11). Notice that $\tau_{q=\pi}$ can diverge for $T \rightarrow 0$ only in the case of antiferromagnetic coupling, $J_{I}<0(\gamma<0)$.

Summarizing, according to the sign of the exchange constant, both time scales $\tau_{q=0}$ (for $J_{I}>0$ ) and $\tau_{q=\pi}$ (for $J_{I}<0$ ) diverge in the low temperature limit $T \rightarrow 0$, following an Arrhenius law

$$
\tau=\frac{1}{2 \alpha} e^{4 \beta\left|J_{I}\right|}
$$

with energy barrier $4\left|J_{I}\right|$ (slow relaxing mode). It is worth noting that the remaining relaxation times, given by the inverse of the eigenvalues in Eq. (11) with $q \neq 0$ and $q \neq \pi$, always remain of the same order of magnitude as $\alpha^{-1}$ (fast relaxing modes). This time scale is typically very small $(\sim$ ps $)$ in real systems, and negligible with respect to the characteristic times involved in any experimental measurement we refer to.

\section{B. The Fourier Transform approach}

The solutions, (16) and (17), to the set of equations (15) can alternatively be deduced within the Fourier Transform (FT) formalism, which has already been exploited to obtain the dispersion relation (11). Recalling the definition (10) of $s_{k}(t)$ and its spatial FT

$$
\widetilde{s}_{q}=\frac{1}{N} \sum_{k} s_{k}(t) e^{-i q k} e^{\lambda_{q} t}
$$

we evaluate $\widetilde{s}_{q}$ at time $t=0, \widetilde{s}_{q}=\frac{1}{N} \sum_{k} s_{k}(0) e^{-i q k}$, for the two initial conditions of interest, (13) and (14). Starting from the all-spin-up configuration, Eq. (13), we have

$$
\widetilde{s}_{q}=\frac{1}{N} \sum_{k} e^{-i q k}=\delta_{q, 0} .
$$

Hence the solution for the expectation value of a spin localized on the $k$ lattice site at time $t$ is

$$
s_{k}(t)=\sum_{q} \delta_{q, 0} e^{i q k} e^{-\lambda_{q} t}=e^{-\lambda_{q=0} t},
$$

which is identical to (16) since $\lambda_{q=0}=\alpha(1-\gamma)$.

Starting from the partially saturated configuration, Eq. (14), it is useful to rewrite it as $s_{k}(0)=-e^{i \pi k}$, so that the FT at $t=0$ is

$$
\widetilde{s}_{q}=-\frac{1}{N} \sum_{k} e^{i \pi k} e^{-i q k}=-\delta_{q, \pi} .
$$

Hence the solution is readily obtained

$$
s_{k}(t)=-\sum_{q} \delta_{q, \pi} e^{i q k} e^{-\lambda_{q} t}=-e^{i \pi k} e^{-\lambda_{q=\pi} t},
$$

which is identical to (17) since $\lambda_{q=\pi}=\alpha(1+\gamma)$.

Finally, we observe that Eqs. (21) and (23) hold even for a ring with a finite number $N$ of spins, while Eqs. (16) and (17) were obtained in the infinite-chain limit.

\section{Slow versus fast relaxation of the spontaneous magnetization}

The expectation values, $s_{k}(t)$, of spins localized on the even and odd sites of a linear lattice at time $t$, computed either with the Generating Functions or the Fourier Transform approach, have been shown to display a mono-exponential relaxation, see Eqs. (16) and (17), with different time scales, $\tau_{q=0}=[\alpha(1-\gamma)]^{-1}$ and $\tau_{q=\pi}[\alpha(1+\gamma)]^{-1}$ respectively, depending on the different initial conditions, Eqs. (13) and (14). As a consequence, also the macroscopic magnetization, expressed by Eq. (4), displays the same mono-exponential relaxation as the single site quantities $s_{k}(t)$.

A chain in which all the magnetic moments are equal can be prepared only in the saturated initial configuration, Eq. (13), with all the spin aligned in the same direction, through the application of an external field. Thus, when the field is abruptly removed, such a system will relax slowly at low temperature only if the exchange coupling is ferromagnetic $\left(J_{I}>0\right)$. In contrast, if the exchange coupling is antiferromagnetic $\left(J_{I}<0\right)$ and the chain is "forced" in the saturated state by a strong applied magnetic field, the system will relax very fast (in a typical time of the order of $\alpha^{-1}$ ) when the field is removed. Let us now discuss how these results, first obtained by Glauber ${ }^{6}$, are generalized to the case of a 
chain in which the magnetic moments are collinear, but not equal on each site.

As pointed out in the introduction, a model with antiferromagnetic coupling $\left(J_{I}<0\right)$ but non-compensated magnetic moments on the two sublattices is more akin to real SCM's $1,3,29$. Yet it is very interesting since, depending on the intensity of the applied magnetic field, the system can be prepared either in the saturated initial configuration, Eq. (13), where all spins are parallel to each other, or in the partially saturated one Eq. (14), where nearest neighbors are antiparallel. In the former case, a very strong field is required in order to overcome the antiferromagnetic coupling between nearest neighbors; once the field is removed, the relaxation of the magnetization is expected to be fast at low temperatures, on the basis of the solution (16). In the latter case, the partially saturated initial configuration (14) can easily be obtained through the application of a smaller, experimentally accessible magnetic field; when the field is abruptly removed, the relaxation is expected to be slow according to the solution (17). The solution (17) justifies the observation of SCM behavior in ferrimagnetic quasi-1D compounds like CoPhOMe ${ }^{29}$ (see Sect. V).

Summarizing, we have found that when a collinear ferrimagnetic chain is prepared in an initial state fully or partially saturated depending on the intensity of the applied magnetic field - once the field is removed abruptly, the spin system can show fast or slow relaxation, respectively. Fast relaxation corresponds to stronger fields; unfortunately for the quasi-1D chain compound $\mathrm{CoPhOMe}{ }^{29,30}$, the antiferromagnetic exchange constant is so large $\left(\left|J_{I}\right| \sim 100 \mathrm{~K}\right)$ that the realization of a fully saturated initial configuration would require a very high, almost unaccessible field ( $1000 \mathrm{kOe})$. Thus this compound is not a good candidate for such a kind of experiments 31 .

\section{MAGNETIC RESPONSE TO AN OSCILLATING MAGNETIC FIELD}

In the presence of a magnetic field $\mathrm{H}$, the transition probability to be put in the equation of motion (5) is $w_{\sigma_{k} \rightarrow-\sigma_{k}}^{\mathrm{H}}$, defined in Eq. (7). One obtains

$$
\begin{aligned}
\frac{d s_{k}(t)}{d t} & =-\alpha\left\{s_{k}(t)-\frac{\gamma}{2}\left[s_{k+1}(t)+s_{k-1}(t)\right]\right. \\
& \left.+\frac{\gamma \delta_{k}}{2}\left[\left\langle\sigma_{k} \sigma_{k+1}\right\rangle_{t}+\left\langle\sigma_{k-1} \sigma_{k}\right\rangle_{t}\right]-\delta_{k}\right\}
\end{aligned}
$$

that differs from Eq. (15), considered earlier for $\mathrm{H}=0$, in the presence of both a non-homogeneous term, $\delta_{k}$, and the time-dependent pair-correlation functions $\left\langle\sigma_{k} \sigma_{k \pm 1}\right\rangle_{t}$. The latter ones, assuming that the field is so weak to induce just small departures from equilibrium, can be approximated by their time-independent counterparts ${ }^{32}$

$$
\left\langle\sigma_{k} \sigma_{k+1}\right\rangle_{t}=\left\langle\sigma_{k-1} \sigma_{k}\right\rangle_{t} \approx \tanh \left(\beta J_{I}\right) \equiv \eta
$$

As it is usual in a.c. susceptibility measurements, we also assume the time-dependent magnetic field $\mathbf{H}(t)=\mathrm{H} e^{-i \omega t} \hat{\mathbf{e}}_{\mathrm{H}}$, oscillating at frequency $\omega$, to be weak so that the $\delta_{k}$ parameters can be linearized

$$
\delta_{k}=\tanh \left(\beta \mu_{B} G_{k} \mathrm{H}(t)\right) \approx \beta \mu_{B} G_{k} \mathrm{H}(t) .
$$

The system of equations of motion (24) then takes the form

$$
\begin{aligned}
\frac{d s_{k}(t)}{d(\alpha t)} & =-s_{k}(t)+\frac{\gamma}{2}\left[s_{k+1}(t)+s_{k-1}(t)\right] \\
& +\beta f\left(\beta J_{I}\right) \mu_{B} G_{k} \mathrm{H}(t)
\end{aligned}
$$

where

$$
f\left(\beta J_{I}\right)=1-\gamma \eta=\frac{1-\eta^{2}}{1+\eta^{2}}
$$

is a function of the reduced coupling constant $\beta J_{I}$ and we have taken into account that $\gamma=2 \eta /\left(1+\eta^{2}\right)$. After a brief transient period, the system will reach the stationary condition in which the magnetic moment of each spin oscillates coherently with the forcing term at the frequency $\omega$. Expressing the expectation value of a spin on the $k$-th lattice site, $s_{k}(t)$, through its spatial FT, $\widetilde{s}_{q}$, the trial solution is

$$
s_{k}(t)=\sum_{q} \widetilde{s}_{q} e^{i q k} e^{-i \omega t} .
$$

Substituting the latter in the system (27) we get

$$
\widetilde{s}_{q}=\beta f\left(\beta J_{I}\right) \mu_{B} \mathrm{H} \frac{\alpha \widetilde{G}_{q}}{\alpha(1-\gamma \cos q)-i \omega},
$$

where $\widetilde{G}_{q}$ is the FT of $G_{k}$ :

$$
\widetilde{G}_{q}=\frac{1}{N} \sum_{k=1}^{N} e^{-i q k} G_{k}
$$

The average of stochastic magnetization can readily be obtained from (4) as

$$
\langle M\rangle_{t}=\mu_{B} e^{-i \omega t} \sum_{k=1}^{N} \sum_{q q^{\prime}} \widetilde{G}_{q} \widetilde{s}_{q^{\prime}} e^{i q k} e^{i q^{\prime} k}
$$

which accounts for non-collinearity of local anisotropy axes with respect to the field direction. Performing the sum over all the lattice sites ( $k$ indices) yields a factor $N \delta_{q,-q^{\prime}}$ in Eq. (32); substituting the expression (30) for $\widetilde{s}_{q}$, one obtains

$$
\langle M\rangle_{t}=N \mu_{B}^{2} \beta f\left(\beta J_{I}\right) \mathrm{H} e^{-i \omega t} \sum_{q q^{\prime}} \frac{\alpha \widetilde{G}_{q} \widetilde{G}_{q^{\prime}} \delta_{q,-q^{\prime}}}{\alpha(1-\gamma \cos q)-i \omega} .
$$

Then, considering that $\widetilde{G}_{q} \widetilde{G}_{-q}=\left|\widetilde{G}_{q}\right|^{2}$, the a.c. susceptibility is finally obtained dividing (33) by $\mathrm{He} e^{-i \omega t}$

$$
\chi(\omega, T)=N \mu_{B}^{2} \beta f\left(\beta J_{I}\right) \sum_{q} \frac{\alpha\left|\widetilde{G}_{q}\right|^{2}}{\alpha(1-\gamma \cos q)-i \omega} .
$$



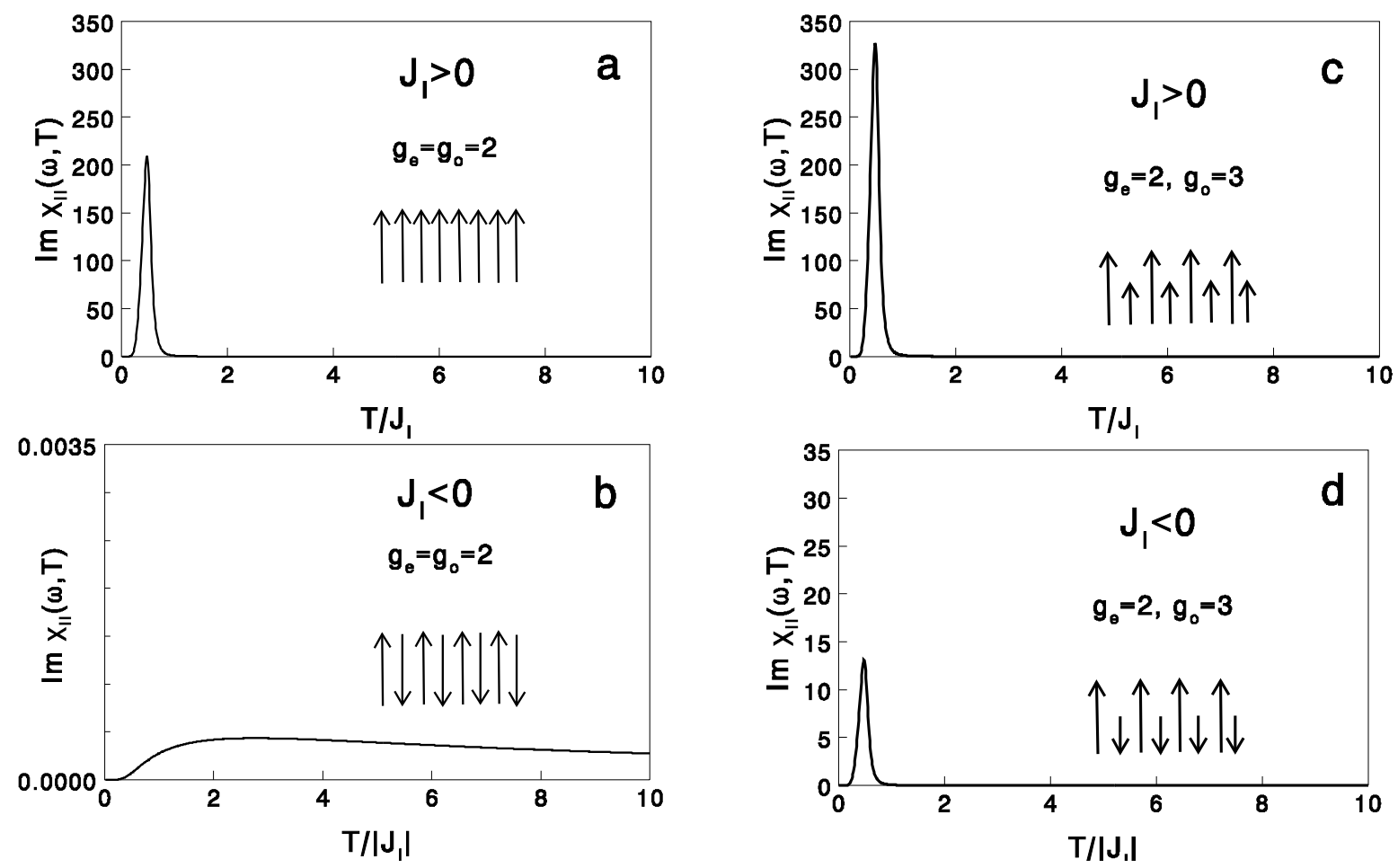

FIG. 1: Temperature dependence of the imaginary part of the complex susceptibility, Eq. (38), of a collinear one-dimensional Ising model with alternating spins. Resonant behavior in response to an oscillating magnetic field is possible, at low frequency, only when magnetic moments are uncompensated (a,c,d), while a broad peak is found when the net magnetization is zero (b). (The curves refer to reduced frequency $\omega / \alpha=0.001$ )

In principle, the a.c. susceptibility of a chain with $N$ spins admits $N$ poles, corresponding to the $N$ eigenvalues $\lambda_{q}$ in Eq. (11). Each mode is related to a different time scale $\tau_{q}=1 / \lambda_{q}$. In practice, not all the time scales will be involved in the complex susceptibility $\chi(\omega, T)$, but only the ones selected by $\widetilde{G}_{q}$. A result similar, at first glance, to Eq. (34) was deduced by Suzuki and $\mathrm{Kubo}^{27}$, but in their case the relationship was between the time scale $\tau_{q}$ and the wave-vector-dependent susceptibility $\chi(q, \omega)$. In contrast, in an a.c. susceptibility experiment only the zero-wave-vector susceptibility $\chi(q=0, \omega)$ is accessible; the peculiarity of Eq. (34) is that other time scales, different from $\tau_{q=0}$, can be selected thanks to the dependence of the gyromagnetic factors $G_{k}$ and of the local anisotropy axes on the site position $k$. This is the main result of our study and will be clarified hereafter through a few examples.

\section{A. The a.c. susceptibility of a collinear Ising ferrimagnetic chain}

Let us start considering the case of a one-dimensional Ising model with two kinds of spins (aligned parallel on antiparallel to the chain axis) alternating on the odd and even magnetic sites of the lattice with Landé fac- tors $G_{2 r+1}=g_{o}$ and $G_{2 r}=g_{e}$ (integer $r$ ), respectively. Strictly speaking, a collinear Ising ferrimagnet is characterized by an antiferromagnetic coupling $J_{I}<0$, but also the case $J_{I}>0$ can be treated through Eq. (34). In fact, since the local axis of anisotropy has the same direction for all the spins, the FT of the site-dependent Landé factor is

$$
\begin{aligned}
\widetilde{G}_{q} & =\frac{1}{N} \sum_{r=1}^{N / 2}\left[g_{e} e^{-i q 2 r}+g_{o} e^{-i q(2 r-1)}\right] \\
& =\left(g_{e}+e^{i q} g_{o}\right) \frac{1}{N} \sum_{r=1}^{N / 2} e^{-i q 2 r}
\end{aligned}
$$

Taking into account that, in the presence of periodic boundary conditions, one has

$$
\sum_{r=1}^{N / 2} e^{-i q 2 r}=\frac{N}{2}\left(\delta_{q, 0}+\delta_{q, \pi}\right),
$$

it follows that the only non-zero values of $\widetilde{G}_{q}$ are for $q=0$ and $q=\pi$

$$
\widetilde{G}_{q}^{\|}=\frac{1}{2}\left[\left(g_{e}+g_{o}\right) \delta_{q, 0}+\left(g_{e}-g_{o}\right) \delta_{q, \pi}\right] .
$$

Thus, according to Eq. (34), the parallel a.c. susceptibility $(\|=z z)$ of a collinear Ising chain with alternating 
spins is

$$
\begin{aligned}
\chi_{\|}(\omega, T) & =N \mu_{B}^{2} \beta f\left(\beta J_{I}\right) \\
& \times \frac{1}{4}\left[\frac{\left(g_{e}+g_{o}\right)^{2}}{(1-\gamma)-i\left(\frac{\omega}{\alpha}\right)}+\frac{\left(g_{e}-g_{o}\right)^{2}}{(1+\gamma)-i\left(\frac{\omega}{\alpha}\right)}\right](38)
\end{aligned}
$$

It appears that both the relaxation times obtained by Suzuki and $\mathrm{Kubo}^{27}$ for the ordinary and the staggered susceptibility of the usual Ising model, namely $\tau_{q=0}=$ $[\alpha(1-\gamma)]^{-1}$ and $\tau_{q=\pi}=[\alpha(1+\gamma)]^{-1}$ respectively, do coexist in the a.c. susceptibility (38). Notice that, in the $\omega \rightarrow 0$ limit, the static susceptibility of the Ising ferrimagnet in zero field ${ }^{17}$ is recovered from Eq. (38), since one has $\frac{f\left(\beta J_{I}\right)}{1 \mp \gamma}=\frac{1-\eta^{2}}{1+\eta^{2}} \frac{1}{1 \mp \gamma}=e^{ \pm 2 \beta J_{I}}$.

As regards the dynamic response of the system to an oscillating magnetic field applied along the chain axis, depending on the sign of the effective exchange coupling constant $J_{I}$, the ferromagnetic $\left(g_{e}+g_{o}\right)$ or the antiferromagnetic $\left(g_{e}-g_{o}\right)$ branch of the parallel susceptibility (38) are characterized by a diverging time scale at low temperature. In particular, for a collinear Ising ferrimagnet one has $J_{I}<0$, so that $\tau_{q=\pi}$ is diverging, while $\tau_{q=0}$ is short (of the order of $\alpha^{-1}$, the attempt frequency of an isolated spin). Thus, for $J_{I}<0$, a resonant behavior of the a.c. susceptibility versus temperature (at low frequencies $\omega / \alpha \ll 1$ ) can only be observed in the case $g_{e} \neq g_{o}$ (see Fig. 1d) when magnetic moments are uncompensated, while a broad peak is found in the case $g_{e}=g_{o}$ when the net magnetization is zero (see Fig. 1b). Clearly, for $J_{I}>0$, a resonant peak is found in both cases (see Fig. 1a and 1c), because a net magnetization is always present in the system.

Such a resonant behavior of the a.c. susceptibility versus $T$, in ferromagnetic ${ }^{33}$ as well as in ferrimagnetic 17 Ising chains with single spin-flip Glauber dynamics, is a manifestation of the stochastic resonance phenomenon ${ }^{34}$ : i.e., the response of a set of coupled bistable systems to a periodic drive is enhanced in the presence of a stochastic noise when a matching occurs between the fluctuationinduced switching rate of the system and the forcing frequency. In a magnetic chain, the role of stochastic noise is played by thermal fluctuations and the resonant peak in the temperature-dependence of the a.c. susceptibility occurs when the statistical time scale, associated to the slow decay of the magnetization, matches with the deterministic time scale of the applied magnetic field

$$
\tau_{q}\left(T_{\text {peak }}\right) \approx \frac{1}{\omega}
$$

\section{B. The a.c. susceptibility of an $n$-fold helix}

Next, as an example of a non-collinear spin arrangement, we consider a system of spins with the local axes of anisotropy arranged on an $n$-fold helix (see Fig. 2); $\theta$ is the angle that the local axes form with $z$, the unique axis of the helix (i.e., the chain axis). In this case the Landé factors are equal on all lattice sites, but different

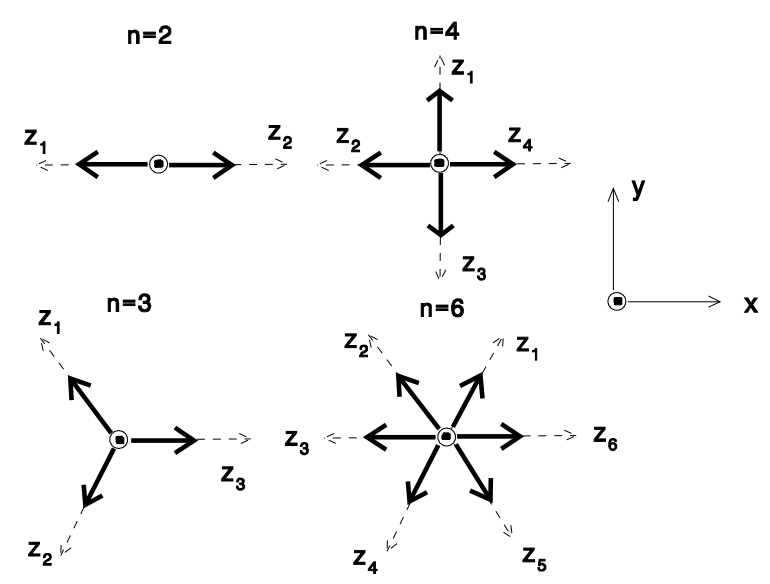

FIG. 2: Thick arrows denote the projections on the $x y$ plane, perpendicular to the chain (helix) axis $z$, of magnetic moments in a one-dimensional Ising helimagnet, for different fold symmetries $(n=2,3,4,6)$. Dashed lines are the projections of the local axes of anisotropy, $\hat{\mathbf{z}}_{k}$.

spins experience different fields because of the geometrical arrangement of magnetic moments. In the following we will make the approximation that the Landé tensor of a spin on the $k$-th lattice site has just a non-zero component $g$ along the easy anisotropy direction $\hat{\mathbf{z}}_{k}$, so that $G_{k}=g \hat{\mathbf{z}}_{k} \cdot \hat{\mathbf{e}}_{\mathrm{H}}$ (see Eq. (3)). In the crystallographic frame $(x, y, z)$, the directors $\hat{\mathbf{z}}_{k}$ read (integer $k$ )

$$
\hat{\mathbf{z}}_{k}=\sin \theta\left[\cos \left(\frac{2 \pi k}{n}\right) \hat{\mathbf{e}}_{x}+\sin \left(\frac{2 \pi k}{n}\right) \hat{\mathbf{e}}_{y}\right]+\cos \theta \hat{\mathbf{e}}_{z} .
$$

Let us first consider the case of an oscillating magnetic field $\mathrm{H}$ applied parallel to $z$, the helix axis. All the spins actually undergo the same field, and since $G_{k}=g \cos \theta$ independently of the lattice site $k$, the only peak in the FT $\widetilde{G}_{q}$ occurs at $q=0$

$$
\widetilde{G}_{q}^{z}=g \cos \theta \delta_{q, 0} \quad(\forall n) .
$$

Following the same procedure as in the previous paragraph, the parallel a.c. susceptibility $(\|=z z)$ takes an expression (valid for any value of the fold index $n$ of the helix)

$$
\chi_{\|}(\omega, T)=N \mu_{B}^{2} \beta f\left(\beta J_{I}\right) \frac{g^{2} \cos ^{2} \theta}{(1-\gamma)-i\left(\frac{\omega}{\alpha}\right)} \quad(\forall n)
$$

that differs from Glauber's result for the collinear Ising chain ${ }^{6}$ only by the geometrical factor $\cos ^{2} \theta$. For ferromagnetic coupling, $J_{I}>0$, the relaxation time $\tau_{0}=$ $[\alpha(1-\gamma)]^{-1}$ diverges as $T \rightarrow 0$, and a resonant behavior of the a.c. parallel susceptibility versus temperature is found, at low frequency, when the oscillating field is applied parallel to the helix axis, $z$, along which spins are uncompensated: see Fig. 3a, which refers to the case of a two-fold helix $(n=2)$. 

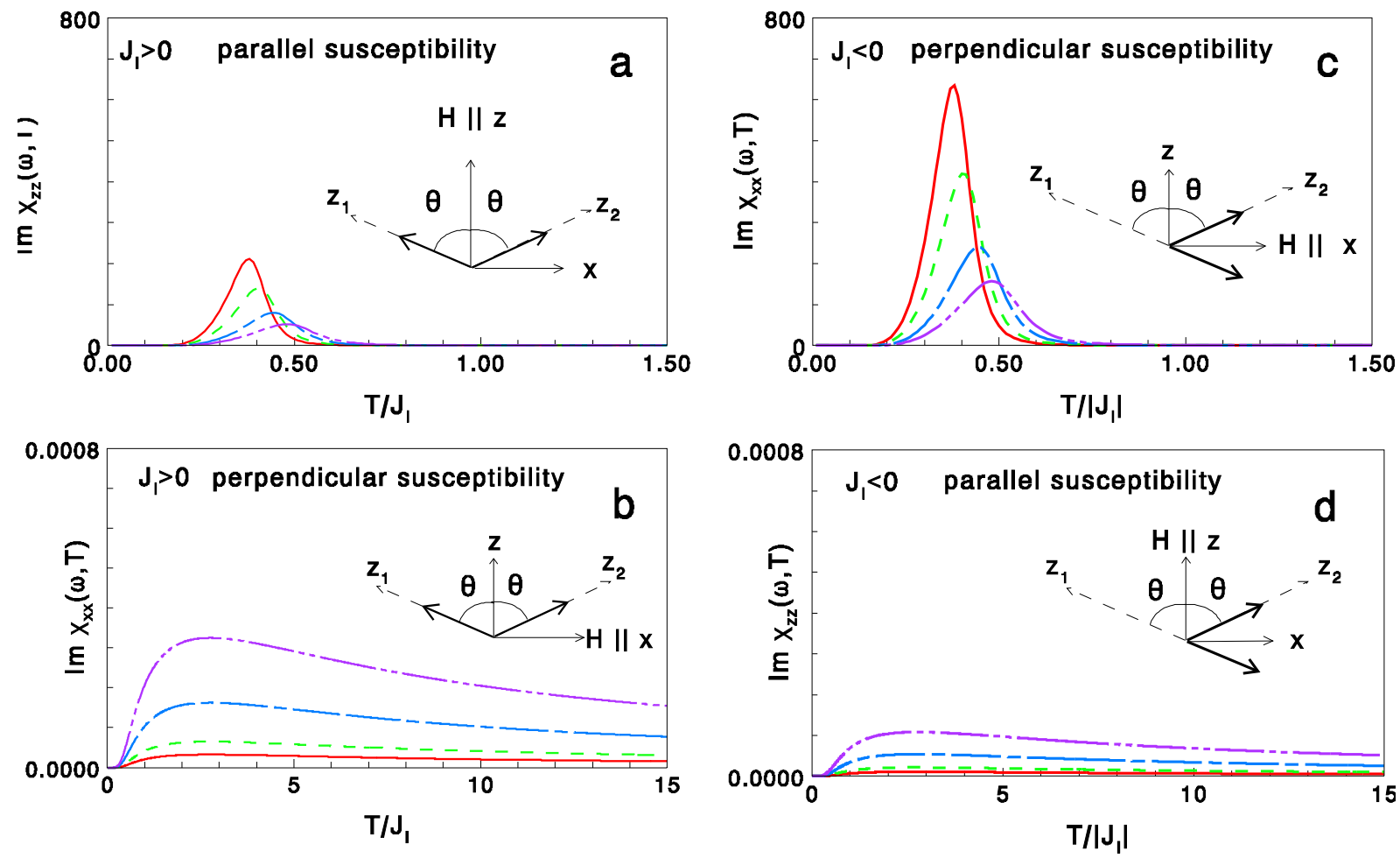

FIG. 3: (color online) Temperature dependence of the imaginary part of the parallel (42) and perpendicular (45) complex susceptibility of an Ising chain with two-fold helical spin arrangement. The local axes $\hat{\mathbf{z}}_{1}$ and $\hat{\mathbf{z}}_{2}$ were assumed to form an angle $\theta=\frac{\pi}{3}$ with $z$, the chain axis (unique axis of the helix). Different curves refer to different values of $\omega / \alpha$ : 0.0001 (continuous, red line); 0.0002 (dashed, green line); 0.0005 (dashed single-dotted, blue line); 0.0010 (dashed double-dotted, violet line). Resonant behavior in response to an oscillating magnetic field is possible, at low frequency, only for field applied in a direction where magnetic moments are uncompensated $(a, c)$, while a broad peak is found $(b, d)$ when there is no net magnetization along the field direction.

Let us now consider the case of an oscillating magnetic field $\mathrm{H}$ applied perpendicularly to the chain axis. In this configuration, it is useful to distinguish the case $n=2$ from the general case $n>2$.

- $n=2$

In this case, it is worth noticing that for $\mathrm{H}$ parallel to $y$, one has identically $G_{r} \equiv 0$ for any lattice site $r$. Thus, $\widetilde{G}_{q}^{y} \equiv 0$ and the corresponding a.c. susceptibility is identically zero

$$
\chi_{y y}(\omega, T) \equiv 0
$$

(not shown). In contrast, for $\mathrm{H}$ parallel to $x$, one has $G_{r}=-g \sin \theta$ on odd sites and $G_{r}=+g \sin \theta$ on even sites. The FT is

$$
\begin{aligned}
\widetilde{G}_{q}^{x} & =\frac{1}{N} \sum_{r=1}^{N / 2} g \sin \theta\left(-e^{-i q(2 r-1)}+e^{-i q 2 r}\right) \\
& =g \sin \theta \frac{1}{2}\left(\delta_{q, 0}+\delta_{q, \pi}\right)\left(1-e^{i q}\right) \\
& =g \sin \theta \delta_{q, \pi}
\end{aligned}
$$

where we have taken into account Eq. (36). Thus, for ferromagnetic coupling, $J_{I}>0$, the relaxation time $\tau_{\pi}=[\alpha(1+\gamma)]^{-1}$ does not diverge as $T \rightarrow 0$, and the perpendicular a.c. susceptibility

$$
\chi_{x x}(\omega, T)=N \mu_{B}^{2} \beta f\left(\beta J_{I}\right) \frac{g^{2} \sin ^{2} \theta}{(1+\gamma)-i\left(\frac{\omega}{\alpha}\right)}
$$

does not present a resonant behavior as a function of temperature; rather, it presents a broad maximum (see Fig. 3b). Clearly, in the case of antiferromagnetic coupling, $J_{I}<0$, the behavior of the susceptibility components is reversed: a broad maximum is found for the temperature dependence of the parallel susceptibility $\chi_{z z}(\omega, T)$ (see Fig. 3d), while a resonant behavior is found for the perpendicular susceptibility $\chi_{x x}(\omega, T)$ (see Fig. 3c).

- $n>2$

In this case, denoting by $\hat{\mathbf{e}}_{x} \cdot \hat{\mathbf{e}}_{\mathrm{H}}$ and $\hat{\mathbf{e}}_{y} \cdot \hat{\mathbf{e}}_{\mathrm{H}}$ the directors of the in-plane field, the FT's of $G_{k}$ are given by

$$
\left\{\begin{aligned}
\widetilde{G}_{q}^{x} & =\frac{1}{N}\left(\hat{\mathbf{e}}_{x} \cdot \hat{\mathbf{e}}_{\mathrm{H}}\right) g \sin \theta \sum_{k=1}^{N} \cos \left(\frac{2 \pi k}{n}\right) e^{-i q k} \\
& =\left(\hat{\mathbf{e}}_{x} \cdot \hat{\mathbf{e}}_{\mathrm{H}}\right) g \sin \theta \frac{1}{2}\left(\delta_{q, \frac{2 \pi}{n}}+\delta_{q,-\frac{2 \pi}{n}}\right) \\
\widetilde{G}_{q}^{y} & =\frac{1}{N}\left(\hat{\mathbf{e}}_{y} \cdot \hat{\mathbf{e}}_{\mathrm{H}}\right) g \sin \theta \sum_{k=1}^{N} \sin \left(\frac{2 \pi k}{n}\right) e^{-i q k} \\
& =\left(\hat{\mathbf{e}}_{y} \cdot \hat{\mathbf{e}}_{\mathrm{H}}\right) g \sin \theta \frac{1}{2 i}\left(\delta_{q, \frac{2 \pi}{n}}-\delta_{q,-\frac{2 \pi}{n}}\right)
\end{aligned}\right.
$$


Remarkably, as just $\left|\widetilde{G}_{q}\right|^{2}$ appears in Eq. (34), the general result for the in-plane susceptibility turns out to be independent of the field direction. Thus, for $n>2$, the perpendicular $(\perp)$ a.c. susceptibility of the $n$-fold helix is given by

$$
\begin{aligned}
& \chi_{\perp}(\omega, T)=N \mu_{B}^{2} \beta f\left(\beta J_{I}\right) \\
\times & \frac{1}{2} \sin ^{2} \theta \frac{g^{2}}{\left[1-\gamma \cos \left(\frac{2 \pi}{n}\right)\right]-i\left(\frac{\omega}{\alpha}\right)},
\end{aligned}
$$

where we have exploited the fact that $\cos \left(-\frac{2 \pi}{n}\right)=$ $\cos \left(\frac{2 \pi}{n}\right)$ for the term appearing at the denominator of Eq. (34).

Summarizing, in the general case of an Ising chain with an $n$-fold helical spin arrangement $(n \geq 2)$, we have explicitly shown that a resonant behavior of the a.c. susceptibility versus temperature, similar to the one displayed by ferromagnetic $\underline{\underline{63}}$ and ferrimagnetic $\frac{17}{1}$ Ising chains with collinear spins, is possible only for field applied in a direction where magnetic moments are uncompensated. In contrast, a broad peak is found when there is no net magnetization along the field direction.

\section{APPLICATION TO REAL SINGLE CHAIN MAGNETS}

In this Section we will apply the developed formalism to some real compounds as representative realizations of SCM's; for the three selected systems - we know this restriction is far from being exhaustive $e^{1.3}-$ a.c. susceptibility data on single crystal are available, which is a fundamental requirement for checking the proposed selection rules. The considered systems $29,35,36$ are characterized by the alternation of two types of magnetic centers along the chain axis, so that at least two spins per cell have to be considered; moreover, the magnetic moments are not collinear, the dominant exchange interactions are antiferromagnetic and a strong single-ion anisotropy is present, which favors magnetization alignment along certain crystallographic directions $\hat{\mathbf{z}}_{k}$. The static properties of these compounds, like magnetization and static susceptibility, are generally well described using a classical Heisenberg model with an isotropic exchange coupling $J$ and a single-ion anisotropy $D$. Thus, in order to describe the dynamic behavior in response to a weak, oscillating magnetic field by means of the previously developed theory, it is necessary to relate the Hamiltonian parameters of such a classical spin model to the exchange constant $J_{I}$ of the effective Ising model (2). In the following we will show, through a few examples on real systems, that indeed, depending on the geometry, selection rules are obeyed for the occurrence of slow relaxation of the magnetization at low temperatures $\left(\beta\left|J_{I}\right| \gg 1\right)$, as well as for resonant behavior of the a.c. susceptibility as a function of temperature at low frequencies. As regards the frequencies involved in an a.c. susceptibility experiment on real SCM's, generally 1,3 they lie in the range
$10^{-1} \div 10^{4} \mathrm{~Hz}$, while the attempt frequency $\alpha$ is of the order of $10^{10} \div 10^{13} \mathrm{~Hz}$. Thus, for a typical experiment, a resonant peak in the a.c. susceptibility can safely be observed provided that at least one of the characteristic time scales $\tau_{q}$ involved in (34) diverges at low $T$, in order for the condition (39) to be satisfied.

\section{A. The $\mathrm{Mn}^{\mathrm{III}}$-based Single Chain Magnet}

In the one-dimensional molecular magnetic compound of formula $\left[\mathrm{Mn}(\mathrm{TPP}) \mathrm{O}_{2} \mathrm{PPhH}\right] \cdot \mathrm{H}_{2} \mathrm{O}$, obtained by reacting $\mathrm{Mn}(\mathrm{III})$ acetate mesotetraphenylporphyrin with phenylphosphinic acid ${ }^{35}$, hereafter denoted by $\mathrm{Mn}^{\mathrm{III}}$ based SCM, the phenylphosphinate anion transmits a sizeable antiferromagnetic exchange interaction that, combined with the easy axis magnetic anisotropy of the $\mathrm{Mn}^{\mathrm{III}}$ sites, gives rise to a canted antiferromagnetic arrangement of the spins. The static single-crystal magnetic properties were analyzed in the framework of a classical spins Hamiltonian

$$
\begin{aligned}
\mathcal{H} & =-\sum_{r=1}^{N / 2}\left\{J \mathbf{S}_{2 r-1} \cdot \mathbf{S}_{2 r}+D\left[\left(S_{2 r-1}^{z_{1}}\right)^{2}+\left(S_{2 r}^{z_{2}}\right)^{2}\right]\right. \\
& \left.+e^{-i \omega t} \mu_{B} \mathrm{H}^{\alpha} g^{\alpha \beta}\left[S_{2 r-1}^{\beta}+S_{2 r}^{\beta}\right]\right\}
\end{aligned}
$$

where $J<0$ is the antiferromagnetic nearest neighbor exchange interaction between $S=2$ spins. $D>0$ is the uniaxial anisotropy favoring two different local axes, alternating along odd and even sites respectively; both axes form an angle $\theta=21.01^{\circ}$ with the crystallographic $c$ axis, while they form opposite angles of modulus $\phi=$ $56.55^{\circ}$ with the $a$ axis (see Fig. 4). Thus we can write $\hat{\mathbf{z}}_{2 r-1}=\sin \theta \cos \phi \hat{\mathbf{e}}_{x}-\sin \theta \sin \phi \hat{\mathbf{e}}_{y}+\cos \theta \hat{\mathbf{e}}_{z}$ and $\hat{\mathbf{z}}_{2 r}=$ $\sin \theta \cos \phi \hat{\mathbf{e}}_{x}+\sin \theta \sin \phi \hat{\mathbf{e}}_{y}+\cos \theta \hat{\mathbf{e}}_{z}$.

A best fit of the static single-crystal magnetic susceptibilities, calculated via a Monte Carlo simulation ${ }^{35}$ provides $J=-1.34 \mathrm{~K}$ and $D=4.7 \mathrm{~K}$; the gyromagnetic tensor $G^{\alpha \beta}$ is diagonal and isotropic with $g^{\|}=1.97$. Equivalent results can be obtained calculating the static properties of model (48) via a transfer matrix approach ${ }^{37}$. Since the uniaxial anisotropy $D$ is rather strong with respect to the exchange coupling $|J|$, as a first approximation one can assume the two sublattice magnetizations to be directed just along the two easy axes, $\hat{\mathbf{z}}_{2 r-1}$ and $\hat{\mathbf{z}}_{2 r}$, so that the chain system (48) can be described by a non-collinear Ising model formally identical to Eq. (2), with an effective ${ }^{38}$ Ising exchange coupling $J_{I}$ and a generalized Landé factor $G_{k}$ defined as, respectively

$$
\begin{aligned}
J_{I} & =J S(S+1) \cos \left(\hat{\mathbf{z}}_{2 r-1} \cdot \hat{\mathbf{z}}_{2 r}\right) \\
G_{r} & =g_{r}^{\|} \sqrt{S(S+1)}\left(\hat{\mathbf{z}}_{r} \cdot \hat{\mathbf{e}}_{\mathrm{H}}\right) .
\end{aligned}
$$

Depending on the orientation of the oscillating magnetic field with respect to the crystallographic axes, the FT of the generalized Landé factor takes the following forms

$$
\widetilde{G}_{q}=g^{\|} \sqrt{S(S+1)}
$$




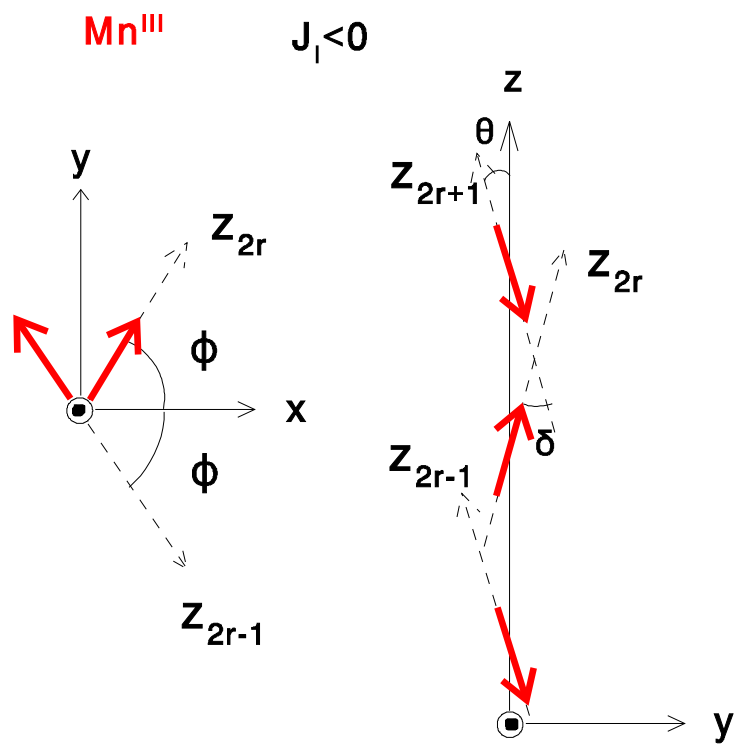

FIG. 4: (color online) Disposition of local axes $\left(\hat{\mathbf{z}}_{2 r-1}\right.$ and $\hat{\mathbf{z}}_{2 r}$ ) and magnetic moments (red arrows) in the $\mathrm{Mn}^{\mathrm{III}}$-based real SCM, discussed in Sect. V.A, with antiferromagnetic effective Ising exchange coupling $J_{I}<0$. Right: Schematic view of the chain structure ( $z$ is the chain axis) along the crystallographic $x$ axis. Left: projections of local axes (dashed lines) and of magnetic moments (red arrows) in the $x y$ plane, perpendicular to the chain axis.

$$
\begin{aligned}
& \times \frac{1}{N} \sum_{r=1}^{N / 2} e^{-i q 2 r}\left[e^{i q}\left(\hat{\mathbf{z}}_{2 r-1} \cdot \hat{\mathbf{e}}_{\mathrm{H}}\right)+\left(\hat{\mathbf{z}}_{2 r} \cdot \hat{\mathbf{e}}_{\mathrm{H}}\right)\right] \\
& =g^{\|} \sqrt{S(S+1)}\left\{\begin{array}{l}
\sin \theta_{1} \cos \phi_{1} \delta_{q, 0}, \mathrm{H} \| x \\
\sin \theta_{1} \sin \phi_{1} \delta_{q, \pi}, \mathrm{H} \| y \\
\cos \theta_{1} \delta_{q, 0}, \mathrm{H} \| z \text { (chain axis) }
\end{array}\right.
\end{aligned}
$$

The corresponding a.c. susceptibility takes the expression

$$
\begin{aligned}
\chi(\omega, T)= & N \mu_{B}^{2} \beta f\left(\beta J_{I}\right)\left(g^{\|}\right)^{2}[S(S+1)] \\
& \times\left\{\begin{array}{l}
\sin ^{2} \theta \cos ^{2} \phi \frac{1}{\left(1-\gamma_{I}\right)-i\left(\frac{\omega}{\alpha}\right)}, \mathrm{H} \| x \\
\sin ^{2} \theta \sin ^{2} \phi \frac{1}{\left(1+\gamma_{I}\right)-i\left(\frac{\omega}{\alpha}\right)}, \mathrm{H} \| y \\
\cos ^{2} \theta \frac{1}{\left(1-\gamma_{I}\right)-i\left(\frac{\omega}{\alpha}\right)}, \mathrm{H} \| z \text { (chain axis) }
\end{array}\right.
\end{aligned}
$$

Taking into account that, for the $\mathrm{Mn}^{\text {III }} \mathrm{SCM}$ under study, the "true" exchange coupling, $J$ in Eq. (48), is antiferromagnetic, and that the angle between the two easy anisotropy axes $\hat{\mathbf{z}}_{1}$ and $\hat{\mathbf{z}}_{2}$ is $\delta=34.6^{\circ}<90^{\circ}$ (see Fig. 4, right), from Eq. (49) it follows that also the effective Ising exchange coupling is antiferromagnetic, $J_{I}<0$. As a consequence, in the low temperature limit $\beta\left|J_{I}\right| \rightarrow \infty$, the relaxation time $\tau_{q=\pi}$ diverges, while $\tau_{q=0}$ does not. Thus, for low frequencies $\omega / \alpha \ll 1$, the a.c. susceptibility presents a resonant behavior only when the oscillating magnetic field is applied along the crystallographic $y$ axis, i.e. the direction, perpendicular to the chain axis, along which the magnetizations of the two sublattices are uncompensated (see Fig. 4). In contrast, when $\mathrm{H}$ is applied parallel to $z$ (the chain axis) or to $x$, namely two directions along which the magnetizations of the two sublattices are exactly compensated, no resonant behavior is expected. These theoretical predictions turn out to be in excellent agreement with experimental a.c. susceptibility data 35 obtained in a single crystal sample of $\left[\mathrm{Mn}(\mathrm{TPP}) \mathrm{O}_{2} \mathrm{PPhH}\right] \cdot \mathrm{H}_{2} \mathrm{O}$, thus confirming that such a $\mathrm{Mn}^{\mathrm{III}}$-based canted antiferromagnet is a bona fide SCM.

\section{B. The Dy ${ }^{\mathrm{II}}$-based Single Chain Magnet}

The molecular magnetic compound of formula $\left[\mathrm{Dy}(\mathrm{hfac})_{3}(\mathrm{NITPhOPh})\right]$, hereafter denoted by $\mathrm{Dy}^{\mathrm{III}}$ based SCM, belongs to a family of quasi one-dimensional magnets in which rare earth ions (with spin $S$ ) and organic radical ions (with $\operatorname{spin} s=1 / 2$ ) alternate themselves along the chain axis, $z$, which in this compound coincides with the crystallographic $b$ axis. Static measurements in single crystal samples suggest ${ }^{36}$ that there is an antiferromagnetic exchange interaction between neighboring Dy ${ }^{\mathrm{III}}$ ions, whose easy anisotropy axes are canted with respect to the chain axis in such a way to generate an uncompensated moment along $b$, while the components in the $a c$ plane are compensated. Thus, as far as the dominant exchange interaction $J<0$ between Dy ${ }^{\mathrm{III}}$ ions is taken into account, the spin Hamiltonian of the system is quite similar to Eq. (48). However, with respect to the $\mathrm{Mn}^{\mathrm{III}}$-based chain, the crystal structure of the Dy ${ }^{\mathrm{III}}$ based SCM is more complicated, not only owing to the presence of two kinds of magnetic centers (the Dy ${ }^{\mathrm{III}}$ ions and the organic radical ions), but mainly because the system is formed by two different families of chains, with two almost orthogonal projections of the easy axes in the $a c$ plane, perpendicular to the chain axis: this "accidental" (in the sense that it is not imposed by symmetry) orthogonality is the reason for the nearly isotropic magnetic behavior displayed by the system within such a plane ${ }^{36}$.

We adopt a simplified model formally equivalent to Eq. (48). Taking into account only the dominant antiferromagnetic exchange interaction $(J<0)$ between neighboring Dy ${ }^{\mathrm{III}}$ ions (which indeed are next nearest neighbors in the real system) and their uniaxial anisotropy $(D>0)$, the system can approximately be described by the classical spins Hamiltonian (48), where now $\left|S_{k}\right|=1$. By means of a classical Transfer Matrix calculation, the static properties of the Dy ${ }^{\mathrm{III}}$-based SCM turn out to be satisfactorily fitted ${ }^{36}$ by $J=-6 \mathrm{~K}, D=40 \mathrm{~K}, g^{\|}=10$, with the two easy anisotropy axes $\hat{\mathbf{z}}_{2 r-1}, \hat{\mathbf{z}}_{2 r}$ forming equal angles $\theta \approx 75^{\circ}$ with the chain axis $z$. (Notice that the latter property holds true for both families of chains.) Also in the case of the Dy ${ }^{I I}$-based SCM, the uniaxial anisotropy $D$ turns out to be sufficiently strong with respect to the exchange coupling $|J|$ in order to assume, 


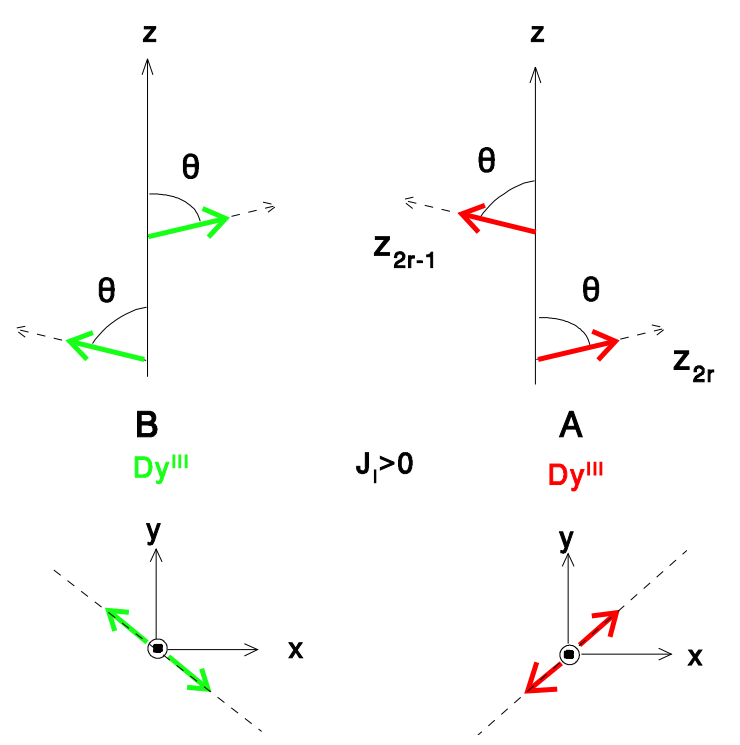

FIG. 5: (color online) Disposition of odd and even local axes $\left(\hat{\mathbf{z}}_{2 r-1}\right.$ and $\left.\hat{\mathbf{z}}_{2 r}\right)$ and magnetic moments (thick arrows) in the Dy ${ }^{\text {III }}$-based real SCM, discussed in Sect. V.B, with ferromagnetic effective Ising exchange coupling $J_{I}>0$. Top: Schematic view of the chain structure ( $z$ is the chain axis), displaying the two families of chains (A, with red magnetic moments, and $\mathrm{B}$, with green magnetic moments). Bottom: projections of magnetic moments in the $x y$ plane, perpendicular to the chain axis.

as a first approximation ${ }^{38}$, the two sublattice magnetizations of Dy ${ }^{I I I}$ to be directed just along the two easy axes. Thus one can define an equivalent non-collinear Ising model (2), where the effective Ising exchange coupling $J_{I}$ and the generalized Landé factor $G_{r}$ are now defined as

$$
\begin{aligned}
J_{I} & =J \cos \left(\hat{\mathbf{z}}_{2 r-1} \cdot \hat{\mathbf{z}}_{2 r}\right) \\
G_{r} & =g_{r}^{\|}\left(\hat{\mathbf{z}}_{r} \cdot \hat{\mathbf{e}}_{\mathrm{H}}\right) .
\end{aligned}
$$

Depending on the orientation of the oscillating magnetic field with respect to the crystallographic axes, the FT of the generalized Landé factor takes the form

$$
\begin{aligned}
\widetilde{G}_{q} & =g^{\|} \frac{1}{N} \sum_{r=1}^{N / 2} e^{-i q 2 r}\left[e^{i q}\left(\hat{\mathbf{z}}_{2 r-1} \cdot \hat{\mathbf{e}}_{\mathrm{H}}\right)+\left(\hat{\mathbf{z}}_{2 r} \cdot \hat{\mathbf{e}}_{\mathrm{H}}\right)\right] \\
& \propto g^{\|} \begin{cases}\cos \theta \delta_{q, 0}, & \mathrm{H} \| z \quad \text { (chain axis) } \\
\sin \theta \delta_{q, \pi}, & \mathrm{H} \perp z\end{cases}
\end{aligned}
$$

It is important to notice that this result holds true for both families $(A, B)$ of chains. Next, we observe that since in the Dy ${ }^{\mathrm{III}}$-based SCM, the spins on opposite sublattices are coplanar with the chain axis, the angle between $\hat{\mathbf{z}}_{2 r-1}$ and $\hat{\mathbf{z}}_{2 r}$ is just $2 \theta \approx 150^{\circ}>90^{\circ}$. Taking into account that the "true" exchange constant in Eq. (48) is antiferromagnetic, $J<0$, from Eq. (52) it follows that the effective Ising exchange coupling is now ferromagnetic, $J_{I}>0$ (see Fig. 5, top). As a consequence, in the low temperature limit $\beta J_{I} \rightarrow \infty$, the relaxation time $\tau_{q=0}$ diverges, while $\tau_{q=\pi}$ does not. Thus, the a.c. susceptibility

$$
\begin{aligned}
\chi(\omega, T) & \propto N \mu_{B}^{2} \beta f\left(\beta J_{I}\right)\left(g^{\|}\right)^{2} \\
& \times \begin{cases}\cos ^{2} \theta \frac{1}{\left(1-\gamma_{I}\right)-i\left(\frac{\omega}{\alpha}\right)}, & \mathrm{H} \| z \text { (chain axis) } \\
\sin ^{2} \theta \frac{1}{\left(1+\gamma_{I}\right)-i\left(\frac{\omega}{\alpha}\right)}, & \mathrm{H} \perp z\end{cases}
\end{aligned}
$$

is expected to have a resonant behavior, for low frequencies $\omega / \alpha \ll 1$, only when the oscillating magnetic field is applied parallel to the chain axis, $z$, along which the magnetizations of the two sublattices are uncompensated (see Fig. 5, top). Such a theoretical prediction turns out to be in excellent agreement with the experimental a.c. susceptibility data ${ }^{36}$ obtained in a single crystal sample of $\left[\mathrm{Dy}(\mathrm{hfac})_{3}(\mathrm{NITPhOPh})\right]_{\infty}$, thus confirming that also the

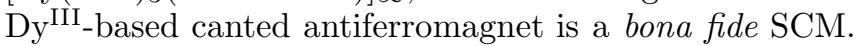
The only qualitative difference, with respect to the $\mathrm{Mn}^{\mathrm{III}}$ based chain is that, due to the different geometry of the spin arrangement and of the local anisotropy axes with respect to the chain axis, the resonant behavior of the a.c. susceptibility is now observed for field applied parallel to the chain axis, rather than perpendicular to it.

\section{The CoPhOMe (Co ${ }^{\mathrm{II}}$-based) Single Chain Magnet}

In the molecular magnetic compound of formula $\left[\mathrm{Co}(\mathrm{hfac})_{2} \mathrm{NITPhOMe}\right]$, hereafter denoted by $\mathrm{CoPhOMe}^{29.30}$, the magnetic contribution is given by Cobalt ions, with an Ising character and effective $S=$ $1 / 2$, and by NITPhOMe organic radical ions, magnetically isotropic and with $s=1 / 2$. The spins are arranged on a helical structure, schematically depicted in Fig. 6, right, whose projections in a plane perpendicular to the helix axis $z$ (coincident with the crystallographic $c$ axis), are represented in Fig. 6, left. The primitive magnetic cell is made up of three Cobalts (black arrows) and three organic radicals (red arrows). Although the effective spins of the two types of magnetic centers have the same value, the gyromagnetic factors are different: $g_{C o} \neq g_{R}$; thus, since the nearest neighbor (Cobalt-radical) exchange interaction is negative (and strong, $|J| \approx 100 \mathrm{~K})^{30}$, the sublattice magnetizations are not compensated along $z$, whereas they are compensated within the $x y$ plane perpendicular to the chain axis $z$. For this compound, which was the first to display SCM behavior $^{29,30}$, static measurements on single-crystal samples has not been interpreted in terms of a simple model yet, due to the complexity of the system itself. Thus, a relationship such as (49) and (52), which associate the Ising Hamiltonian (2) parameters $\left(J_{I}\right.$ and $\left.G_{k}\right)$ with those of a more realistic Hamiltonian, is still missing. However, the dynamic behavior has been thoroughly investigated treating - for the sake of simplicity - both the $\mathrm{Co}^{\mathrm{II}}$ and the organic radical spins as Ising variables, with $\sigma= \pm 1$. 


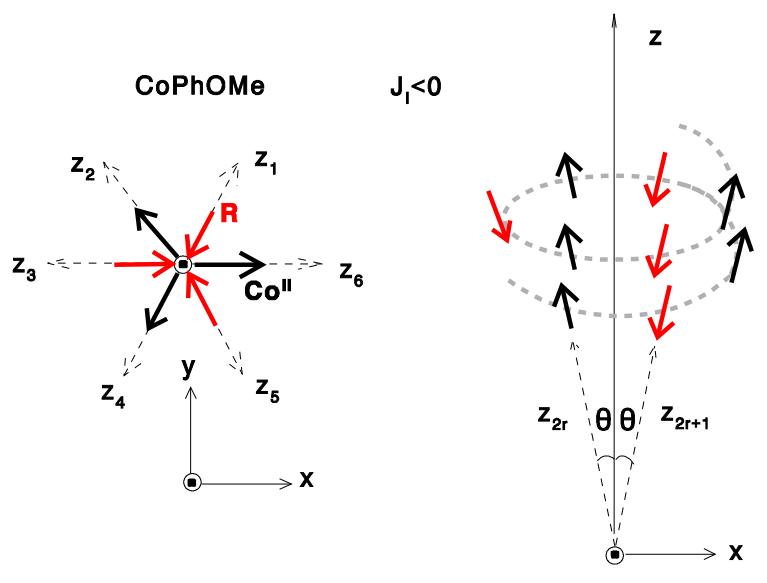

FIG. 6: (color online) Disposition of even and odd local axes (dashed lines) and magnetic moments (thick arrows) in the CoPhOMe real SCM, discussed in Sect. V.C, with antiferromagnetic effective Ising exchange coupling $J_{I}<0$. Right: Schematic view of the chain structure ( $z$ is the chain axis) along the crystallographic $y$ axis. Left: projections of local axes (dashed lines) and of magnetic moments (thick arrows) in the $x y$ plane, perpendicular to the chain axis.

The effective Ising Hamiltonian reads

$$
\begin{aligned}
& \mathcal{H}=-\sum_{l=1}^{N / 6} \sum_{m=1}^{3}\left\{J_{I} \sigma_{l, 2 m}\left[\sigma_{l, 2 m-1}+\sigma_{l, 2 m+1}\right]+e^{-i \omega t} \mu_{B} \mathrm{H}\right. \\
& \left.\left[g_{R} \sigma_{l, 2 m-1}\left(\hat{\mathbf{z}}_{2 m-1} \cdot \hat{\mathbf{e}}_{\mathrm{H}}\right)+g_{C o} \sigma_{l, 2 m}\left(\hat{\mathbf{z}}_{2 m} \cdot \hat{\mathbf{e}}_{\mathrm{H}}\right)\right]\right\}
\end{aligned}
$$

with $l$ magnetic cell index and $m$ site label with boundary conditions $\sigma_{l, 7}=\sigma_{l+1,1}$. Since all the local axes $\hat{\mathbf{z}}_{k}(k=$ $1, \cdots, 6)$ form the same angle $\theta \approx 55^{\circ}$ with the $z$ axis, when a magnetic field is applied along $z$, the FT of the generalized Landé factor is simply given by

$$
\begin{aligned}
& \widetilde{G}_{q}^{\|}=\cos \theta \frac{1}{N} \sum_{r=1}^{N / 2}\left(g_{C o} e^{-i q(2 r-1)}+g_{R} e^{-i q 2 r}\right) \\
& =\frac{\cos \theta}{2}\left[\left(g_{C o}+g_{R}\right) \delta_{q, 0}+\left(g_{C o}-g_{R}\right) \delta_{q, \pi}\right]
\end{aligned}
$$

which, except for the prefactor $\cos \theta$, is quite similar to Eq. (37) for the collinear Ising chain with alternating spins. Thus, the parallel a.c. susceptibility is

$$
\begin{aligned}
\chi_{\|}(\omega, T) & =N \mu_{B}^{2} \beta f\left(\beta J_{I}\right) \frac{\cos ^{2} \theta}{4} \\
& \times\left[\frac{\left(g_{C o}+g_{R}\right)^{2}}{\left(1-\gamma_{I}\right)-i\left(\frac{\omega}{\alpha}\right)}+\frac{\left(g_{C o}-g_{R}\right)^{2}}{\left(1+\gamma_{I}\right)-i\left(\frac{\omega}{\alpha}\right)}\right]
\end{aligned}
$$

Taking into account that the effective exchange coupling of CoPhOMe is negative $\left(J_{I}<0\right)$, the antiferromagnetic branch of the parallel susceptibility is characterized by a diverging time scale $\tau_{q=\pi}=\left[\alpha\left(1+\gamma_{I}\right)\right]^{-1}$ at low temperature, so that, for low frequencies $\omega / \alpha \ll 1, \chi_{\|}(\omega, T)$ displays a resonant behavior.
Let us now consider the case of a field applied in the plane perpendicular to the chain axis. For $\mathrm{H} \| x$ (see Fig. 6 , left) one has, letting $k_{0}=\frac{\pi}{3}$

$$
\begin{aligned}
& G_{2 r-1}^{x}=g_{R} \sin \theta \cos \left[k_{0}(2 r-1)\right] \\
& G_{2 r}^{x}=g_{C o} \sin \theta \cos \left[k_{0} 2 r\right]
\end{aligned}
$$

so that the FT takes the form

$$
\begin{aligned}
\widetilde{G}_{q}^{x} & =\sin \theta \frac{1}{N} \sum_{r=1}^{N / 2} e^{-i q 2 r}\left(g_{C o} \cos \left(k_{0} 2 r\right)\right. \\
& \left.+g_{R} e^{i q}\left[\cos \left(k_{0}\right) \cos \left(k_{0} 2 r\right)+\sin \left(k_{0}\right) \sin \left(k_{0} 2 r\right)\right]\right) \\
& =\frac{1}{4} \sin \theta\left[\left(g_{C o}+g_{R} e^{i\left(q-k_{0}\right)}\right)\left(\delta_{q, k_{0}}+\delta_{q, \pi+k_{0}}\right)\right. \\
& \left.+\left(g_{C o}+g_{R} e^{i\left(q+k_{0}\right)}\right)\left(\delta_{q,-k_{0}}+\delta_{q, \pi-k_{0}}\right)\right]
\end{aligned}
$$

where, as usual, we have exploited Eq. (36). Thus it follows that

$$
\begin{aligned}
& \widetilde{G}_{q= \pm \frac{\pi}{3}}=\frac{\sin \theta}{4}\left(g_{C o}+g_{R}\right) \\
& \widetilde{G}_{q=\pi \pm \frac{\pi}{3}}=\frac{\sin \theta}{4}\left(g_{C o}-g_{R}\right) .
\end{aligned}
$$

The corresponding relaxation times are $\tau_{q= \pm \frac{\pi}{3}}=\frac{\alpha}{1-\frac{1}{2} \gamma}$ and $\tau_{q= \pm \frac{2 \pi}{3}}=\frac{\alpha}{1+\frac{1}{2} \gamma}$ so that, summing the four contributions we obtain the perpendicular a.c. susceptibility

$$
\begin{gathered}
\chi_{\perp}(\omega, T)=N \mu_{B}^{2} \beta f\left(\beta J_{I}\right) \frac{\sin ^{2} \theta}{8} \\
\times\left[\frac{\left(g_{C o}+g_{R}\right)^{2}}{\left(1-\frac{1}{2} \gamma\right)-i\left(\frac{\omega}{\alpha}\right)}+\frac{\left(g_{C o}-g_{R}\right)^{2}}{\left(1+\frac{1}{2} \gamma\right)-i\left(\frac{\omega}{\alpha}\right)}\right] .
\end{gathered}
$$

In conclusion, for the six-fold helix model with alternating spins and Ising exchange coupling in Eq. (55), the parallel and perpendicular components of the a.c. susceptibility, $\chi_{\|}(\omega, T)$ and $\chi_{\perp}(\omega, T)$, display a behavior similar to that of a ferrimagnetic chain with alternating spins (see (38) ) and of an $n$-fold helical spin arrangement with equivalent spins (see (47)), respectively. In spite of the approximations involved in model (55) to describe the real $\mathrm{CoPhOMe}$ molecular magnetic chain, the two calculated susceptibilities (57) and (61), qualitatively reproduce the dynamic behavior of this compound ${ }^{29,30}$. In fact, no out-of-phase a.c. susceptibility (imaginary part) is observed when the field is applied in the plane perpendicular to the chain axis, $z$, for the experimental frequencies $\left(1 \div 10^{5} \mathrm{~Hz}\right)^{30}$. In contrast, when the oscillating field is parallel to $z$, a resonant behavior is observed as a function of temperature. Even though our theoretical treatment holds only for small deviations from equilibrium, it is worth mentioning that the absence of slow relaxation for fields applied in the perpendicular plane is evidenced in the low temperature magnetization curve as well: at low enough temperatures, a finite-area hysteresis loop is present only when a static field is applied parallel to the chain axis, while no hysteresis is observed in the in-plane magnetization curve ${ }^{29,30}$. 


\section{CONCLUSIONS}

In conclusion, in the framework of a one-dimensional Ising model with single spin-flip Glauber dynamics, taking into account reciprocal non-collinearity of local anisotropy axes and the crystallographic (laboratory) frame, we have investigated: (i) the dynamics of magnetization reversal in zero field, and (ii) the response of the system to a weak magnetic field, oscillating in time. We have shown that SCM behavior is not only a feature of collinear ferro- and ferrimagnetic, but also of canted antiferromagnetic chains. In particular, we have found that resonant behavior of the a.c. susceptibility versus temperature in response to an oscillating magnetic field is possible, at low frequency, only for fields applied in a direction where magnetic moments are uncompensated. In contrast, a broad peak is expected when there is no net magnetization along the field direction.

The role played by geometry in selecting the time scales involved in a process is an important and peculiar result, typical of magneto-molecular approach to lowdimensional magnetism. In fact, magnetic centers with uniaxial anisotropy usually correspond to building blocks with low symmetry ${ }^{39,40}$, which - in turn - often crystallize in more symmetric space groups, realizing a reciprocal non-collinearity between local anisotropy axes as a natural consequence ${ }^{1,2}$. Thus the family of real SCM's, to which our model applies, does not restrict to ad-hoc synthesized compounds but, instead, is expected to grow larger in the future ${ }^{3}$. As a validity check of our selection rules (as well as a tutorial exemplification), we have shown how our theory applies successfully to three different molecular-based spin chains; when possible, we have put the parameters of our model Hamiltonian (2) in relationship with those of more general models, typically used to fit the static properties of the corresponding compounds. Needless to say that the possibility of schematizing the chosen three compounds with Hamiltonian (2) relies on the fact that at low enough temperatures they behave as chains consisting of two-level units coupled by a fully anisotropic exchange interaction. The latter assumption is expected to hold also for spin larger than one-half in the presence of strong single ion anisotropy, provided that domain walls still remain sharp 24,38 . In this case each single magnetic center follows a thermally activated dynamics, with an energy barrier $\Delta_{0}$, and well established heuristic arguments ${ }^{41}$ suggest to replace the attempt frequency by $\alpha=\alpha_{0} e^{-\beta \Delta_{0}}$.

A naive application of our 3 -fold-helix results (42) and (47) to the recently synthesized non-collinear $\mathrm{Dy}_{3}$ cluster would prevent the observation of slow relaxation, while Single-Molecule-Magnet dynamics is indeed there observed even in the presence of compensated magnetic moments ${ }^{42}$. However, such a behavior in the classical regime, i.e. far from level crossings where underbarrier processes of quantum origin are important, is observed for $\mathrm{Dy}_{3}$ in non-zero field and the resonant behavior is due to a change of the relative population between the lowest and the first excited Kramers doublets of each Dy ion: For sure this mechanism cannot be accounted for when dealing with two-level elementary variables, like $\sigma_{k}$ in Hamiltonian (2). An extension of our model to multivalued $\sigma_{k}$ 's definitely deserve to be considered in the next future.

Beyond molecular spin chains, our approach might also be used to model monatomic nanowires showing slow relaxation of the magnetization at low temperatures 43 and, possibly, one-dimensional spin glasses 44 (provided that quenched disorder is somehow taken into account). In this regard, the question of distinguishing between SCM and spin-glass behavior in quasi-1D systems is still a hot topic of discussion $45,46,47,48$.

After the successful organization of Single-Molecule Magnets onto surfaces $49,50,51$, the grafting of properly functionalized SCM's on substrates represents a foreseeable goal as well as a fundamental step for their possible use as magnetic-memory units ${ }^{3}$. Technologies employing more traditional materials but based on alternative geometrical arrangement of magnetic anisotropy axes with respect to the switching field, such as in perpendicular recording 52 or processional switching 53 , are already at the stage of forthcoming implementation in devices. Were SCM's to be considered as a possible route to tackle the main issues of high-density magnetic storage - i.e. optimization of the signal-noise ratio, thermal stability and writability 52 - the proposed selection rules for slow relaxation, and related bistability, might find an application in magnetic-memory manufacture as well.

\section{Acknowledgments}

We wish to thank R. Sessoli and A. Rettori for stimulating discussions, and J. Villain for interest and fruitful suggestions in the early stages of this research work. Financial support from ETHZ, the Swiss National Foundation, and Italian National Research Council is also acknowledged.

\section{APPENDIX A: THE GENERATING FUNCTIONS APPROACH}

Assuming periodic boundary conditions and defining the two generating functions

$$
\begin{aligned}
& \mathcal{L}(y, t)=\sum_{r=-\infty}^{+\infty} y^{2 r+1} s_{2 r+1}(t) \\
& \mathcal{G}(y, t)=\sum_{r=-\infty}^{+\infty} y^{2 r} s_{2 r}(t),
\end{aligned}
$$

Eqs. (15) take the form of two differential equations (with the dot indicating the first derivative with respect to the 
adimensional variable $\alpha t$ )

$$
\left\{\begin{array}{l}
\dot{\mathcal{L}}(y, t)=-\mathcal{L}(y, t)+\frac{1}{2} \gamma\left(y+y^{-1}\right) \mathcal{G}(y, t) \\
\dot{\mathcal{G}}(y, t)=-\mathcal{G}(y, t)+\frac{1}{2} \gamma\left(y+y^{-1}\right) \mathcal{L}(y, t)
\end{array}\right.
$$

The system (A2) can be decoupled through the substitution

$$
\begin{aligned}
\mathcal{U}(y, t) & =\mathcal{L}(y, t)+\mathcal{G}(y, t) \\
\mathcal{W}(y, t) & =\mathcal{L}(y, t)-\mathcal{G}(y, t),
\end{aligned}
$$

from which we directly get

$$
\left\{\begin{array}{l}
\dot{\mathcal{U}}(y, t)=-(1-\nu) \mathcal{U}(y, t) \\
\dot{\mathcal{W}}(y, t)=-(1+\nu) \mathcal{W}(y, t)
\end{array}\right.
$$

with $\nu=\frac{1}{2} \gamma\left(y+y^{-1}\right)$. The solutions of these two equations are $\mathcal{U}(y, t)=\mathcal{U}(y, 0) e^{-(1-\nu) \alpha t}$ and $\mathcal{W}(y, t)=$ $\mathcal{W}(y, 0) e^{-(1+\nu) \alpha t}$ that, exploiting the property

$$
\exp \left[\frac{1}{2}\left(y+y^{-1}\right) x\right]=\sum_{k=-\infty}^{+\infty} y^{k} \mathcal{I}_{k}(x)
$$

of the Bessel functions of imaginary argument $\mathcal{I}_{k}(x)$, can be rewritten as

$$
\left\{\begin{array}{l}
\mathcal{U}(y, t)=\mathcal{U}(y, 0) e^{-\alpha t} \sum_{k=-\infty}^{+\infty} y^{k} \mathcal{I}_{k}(\gamma \alpha t) \\
\mathcal{W}(y, t)=\mathcal{W}(y, 0) e^{-\alpha t} \sum_{k=-\infty}^{+\infty}(-1)^{k} y^{k} \mathcal{I}_{k}(\gamma \alpha t)
\end{array}\right.
$$

Performing the inverse transformation of (A3), the solutions to the system (A2) can be obtained

$$
\begin{aligned}
& \mathcal{L}(y, t)=\frac{1}{2} e^{-\alpha t} \times \\
& \sum_{k=-\infty}^{+\infty} y^{k}\left[\mathcal{U}(y, 0) \mathcal{I}_{k}(\gamma \alpha t)+(-1)^{k} \mathcal{W}(y, 0) \mathcal{I}_{k}(\gamma \alpha t)\right] \\
& \mathcal{G}(y, t)=\frac{1}{2} e^{-\alpha t} \times \\
& \sum_{k=-\infty}^{+\infty} y^{k}\left[\mathcal{U}(y, 0) \mathcal{I}_{k}(\gamma \alpha t)-(-1)^{k} \mathcal{W}(y, 0) \mathcal{I}_{k}(\gamma \alpha t)\right] .
\end{aligned}
$$

Then, separating the $k$-odd from the $k$-even terms in both sums, we get

$$
\mathcal{L}(y, t)=e^{-\alpha t} \sum_{r=-\infty}^{+\infty}\left[y^{2 r} \mathcal{L}(y, 0) \mathcal{I}_{2 r}(\gamma \alpha t)\right.
$$

* Electronic address: vindigni@phys.ethz.ch

1 C. Coulon, H. Miyasaka, and R. Clérac, Struct. Bond. 122, 163 (2006), and references therein.

${ }^{2}$ H. Miyasaka and M. Yamashita, Dalton Trans., 399 (2007), and references therein.

$$
\begin{aligned}
& \left.+y^{2 r+1} \mathcal{G}(y, 0) \mathcal{I}_{2 r+1}(\gamma \alpha t)\right] \\
& \mathcal{G}(y, t)=e^{-\alpha t} \sum_{r=-\infty}^{+\infty}\left[y^{2 r} \mathcal{G}(y, 0) \mathcal{I}_{2 r}(\gamma \alpha t)\right. \\
& \left.+y^{2 r-1} \mathcal{L}(y, 0) \mathcal{I}_{2 r-1}(\gamma \alpha t)\right] \text {. }
\end{aligned}
$$

According to A1 , now $\mathcal{L}(y, 0)$ and $\mathcal{G}(y, 0)$ can be expressed again in terms of the initial single spin expectation values $s_{2 r}(0)$ and $s_{2 r+1}(0)$ respectively

$$
\begin{aligned}
& \mathcal{L}(y, t)=e^{-\alpha t} \sum_{r=-\infty}^{+\infty} \\
& \times\left[y^{2 r} \sum_{m=-\infty}^{+\infty} y^{2 m+1} s_{2 m+1}(0) \mathcal{I}_{2 r}(\gamma \alpha t)\right. \\
& \left.+y^{2 r+1} \sum_{m=-\infty}^{+\infty} y^{2 m} s_{2 m}(0) \mathcal{I}_{2 r+1}(\gamma \alpha t)\right]
\end{aligned}
$$

Putting $k^{\prime}=k+m$ we have

$$
\begin{aligned}
& \left.\mathcal{L}(y, t)=e^{-\alpha t} \sum_{\substack{k^{\prime}=-\infty \\
+\infty}}^{+\infty} y^{2 k^{\prime}+1} \sum_{m=-\infty}^{+\infty}(\gamma \alpha t)+s_{2 m}(0) \mathcal{I}_{2\left(k^{\prime}-m\right)+1}(\gamma \alpha t)\right] . \\
& {\left[s_{2 m+1}(0) \mathcal{I}_{2\left(k^{\prime}-m\right)}(\gamma \alpha\right.}
\end{aligned}
$$

Comparing this latter result with the definition of $\mathcal{L}(y, t)$ (A1) and requiring for the terms corresponding to the same power of $y$ to be equal, an explicit function for the odd spin expectation values is readily obtained

$$
\begin{aligned}
& s_{2 r+1}(t)=e^{-\alpha t} \sum_{m=-\infty}^{+\infty}\left[s_{2 m+1}(0) \mathcal{I}_{2(r-m)}(\gamma \alpha t)\right. \\
& \left.+s_{2 m}(0) \mathcal{I}_{2(r-m)+1}(\gamma \alpha t)\right] \text {. }
\end{aligned}
$$

Substituting $\mathcal{L}(y, 0)$ e $\mathcal{G}(y, 0)$ in the solution found for $\mathcal{G}(y, t)$ and performing similar passages, we obtain the expectation value for even sites

$$
\begin{aligned}
& s_{2 r}(t)=e^{-\alpha t} \sum_{m=-\infty}^{+\infty}\left[s_{2 m}(0) \mathcal{I}_{2(r-m)}(\gamma \alpha t)\right. \\
& \left.+s_{2 m+1}(0) \mathcal{I}_{2(r-m)-1}(\gamma \alpha t)\right] .
\end{aligned}
$$

${ }^{3}$ L. Bogani et al., J. Mater. Chem. 18, 4750 (2008), and references therein.

4 H. B. Braun, Phys. Rev. Lett. 71, 3557 (1993).

${ }^{5}$ H. B. Braun, J. Appl. Phys. 85, 6172 (1999), and references therein. 
${ }^{6}$ R. J. Glauber, J. Math. Phys. 4, 294 (1963).

7 J. E. Anderson, J. Chem. Phys. 52, 2021 (1969).

8 S. Bozdemir, Phys. Status Solidi B 103, 459 (1981).

9 S. Bozdemir, Phys. Status Solidi B 104, 37 (1980).

10 J. L. Skinner, J. Chem. Phys. 79, 1955 (1983).

11 G. O. Berim and E. Ruckenstein, J. Chem. Phys. 119, 9640 (2003).

12 J. J. Brey and A. Prados, Phys. Rev. E 53, 458 (1996).

13 R. Cordery, S. Sarker, and J. Toboshnik, Phys. Rev. B 24, 5402 (1981).

14 J. Kamphorst Leal da Silva, A. G. Moreira, M. S. Soares, and F. C. Sá Barreto, Phys. Rev. E 52, 4527 (1995).

15 R. B. Stinchcombe, J. E. Santos and M. D. Grynberg, J. Phys. A 31, 541 (1998).

16 M. Droz, J. K. L. da Silva and A. Malaspinas, Phys. Lett. A 115, 448 (1986).

17 M. G. Pini and A. Rettori, Phys. Rev. B 76, 064407 (2007) [Erratum: Phys. Rev. B 76, e069903 (2007)].

18 E. Ising, Z. Phys. 31, 253 (1925).

19 M. Einax and M. Schulz, J. Chem. Phys. 115, 2282 (2001).

20 J. B. Goodenough, Magnetism and the Chemical Bond, Interscience, New York, 1963.

21 J. B. Goodenough, J. Phys. Chem. Solids 6, 287 (1958).

22 J. Kanamori, J. Phys. Chem. Solids 10, 87 (1959).

${ }^{23}$ L. Lecren et al., J. Am. Chem. Soc. 129, 5045 (2007).

24 A. Vindigni, Inorg. Chim. Acta 361, 3731 (2008).

25 B. U. Felderhof and M. Suzuki, Physica 56, 43 (1971).

26 J. C. Kimball, J. Stat. Phys. 21, 289 (1979).

27 M. Suzuki, R. Kubo, J. Phys. Soc. Japan 24, 51 (1968).

28 J. H. Luscombe, M. Luban, and J. P. Reynolds, Phys. Rev. E 53, 5852 (1996).

29 A. Caneschi et al., Angew. Chem. Int. Ed. Ingl. 40, 1790 (2001).

30 A. Caneschi et al., Europhys. Lett. 58, 771 (2002).

31 It is worth noticing that, once a good candidate were found, an experimental verification of the theoretical prediction might be not so easy since the considered relaxation is a strongly out-of-equilibrium process, while in our theory small departures from equilibrium were assumed.

${ }^{32}$ K. Huang, Statistical Mechanics, J. Wiley and C., New York, 1987.

33 J. J. Brey and A. Prados, Phys. Lett. A 216, 240 (1996).
34 L. Gammaitoni, P. Hänggi, P. Jung and F. Marchesoni, Rev. Mod. Phys. 70, 223 (1998).

${ }^{35}$ K. Bernot et al., J. Am. Chem. Soc. 130, 1619 (2008).

${ }^{36} \mathrm{~K}$. Bernot, Lanthanides in molecular magnetism: from mononuclear Single Molecule Magnets to Single Chain Magnets, Ph.D. thesis, INSA-Rennes, France (November 2007).

37 R. Pandit and C. Tannous, Phys. Rev. B 28, 281 (1983).

38 For a collinear Heisenberg ferromagnet with exchange $J$ and anisotropy $D$, the energy cost of a domain wall was calculated and compared with the energies of a sharp wall and of a soliton, and a the crossover between the "sharp wall" regime $(J \ll D)$ and the "broad wall" regime $(J \gg$ $D)$ was found to occur ${ }^{24}$ for $J / D=1.8$. In principle, a similar calculation should be performed also for the noncollinear model (48), in order to find the limits of validity for the approximation made in Eq. (49).

39 D. Gatteschi et al., Science 265, 1054 (1994).

40 D. Gatteschi and R. Sessoli, Angew. Chem. Int. Ed. 42, 268 (2003), and references therein.

41 C. Coulon, R. Clérac1, L. Lecren, W. Wernsdorfer, and H. Miyasaka, Phys. Rev. B 69, 132408 (2004).

42 J. Luzon et al., Phys. Rev. Lett. 100, 247205 (2008).

43 P. Gambardella et al., Nature 416, 301 (2002).

44 J. A. Mydosh, Spin Glasses: An Experimental Introduction, Taylor and Francis Ltd., London, 1993.

45 A. Maignan et al., Eur. Phys. J. B 15, 657 (2000).

46 S. J. Etzkorn, W. Hibbs, J. S. Miller, and A. J. Epstein, Phys. Rev. B 70, 134419 (2004).

47 L. Bogani, Magnetic and magneto-optical properties of molecular compounds, Ph.D. thesis, Dipartimento di Chimica, Università di Firenze, Italy (December 2005).

48 M.A. Girtu et al., J. Appl. Phys., 81, 4410 (1997).

49 A. Cornia et al., Angew. Chem. Int. Ed. Ingl., 42, 1645 (2003).

50 A. N. Abdi et al., J. Appl. Phys. 95, 7345 (2004).

51 M. Mannini et al., Chem. Eur. J. 14, 7530 (2008).

52 S. N. Piramanayagam and K. Srinivasan, J. Mag. Mag. Mat., in press (2008).

${ }^{53}$ C. H. Back et al., Science 285, 864 (1999). 